\title{
1 Multi-area recordings and optogenetics 2 in the awake, behaving marmoset
}

3 Patrick Jendritza ${ }^{1,2}$, Frederike J. Klein ${ }^{1}$ and Pascal Fries ${ }^{1,2,3}$

4 1. Ernst Strüngmann Institute (ESI) for Neuroscience in Cooperation with Max Planck Society, Frankfurt, Germany

5 2. International Max Planck Research School for Neural Circuits, Frankfurt, Germany

6 3. Donders Institute for Brain, Cognition and Behaviour, Radboud University Nijmegen, Nijmegen, Netherlands

Correspondence: patrick.jendritza@esi-frankfurt.de

8 Abstract

9 The common marmoset has emerged as a key primate model in neuroscience. Marmosets are small in size, show great potential as transgenic models and exhibit complex behaviors. These advantages place the marmoset model in the critical gap between rodents and larger primates. Thus, it is necessary to develop technology that enables monitoring and manipulation of the neural circuits underlying the behavior of the marmoset. Here, we present a novel approach to record and optogenetically manipulate neural activity in the awake, behaving marmoset. Our design utilizes a light-weight, 3D printed titanium chamber that can house several high-density silicon probes for semi-chronic recordings, while enabling simultaneous optogenetic stimulation.

17 Surgical procedures are streamlined via custom 3D printed guides and implantation holders. We demonstrate the application of our method by recording multi- and single-unit data from areas $\mathrm{V} 1$ and V6 with 192 channels simultaneously, and show for the first time that optogenetic activation of excitatory neurons in area V6 can influence behavior in a detection task. Together, the work

21 presented here will support future studies investigating the neural basis of perception and 22 behavior in the marmoset. 


\section{Introduction}

24 The common marmoset (Callithrix jacchus) is becoming an important animal model in

25 neuroscience (Solomon and Rosa, 2014; Miller, 2017; Servick, 2018; Okano, 2021). Due to its

26 small size, genetic tractability (Sasaki et al., 2009; Tomioka et al., 2017; Sato et al., 2020) and

27 complex behavioral repertoire (Stevenson and Poole, 1976; Mitchell and Leopold, 2015; Miller et al., 2016), it is placed in the critical gap between rodent models and larger primate models. Thus, marmosets hold great potential for improving our understanding of the neural circuits underlying complex behaviors and perception. It is therefore pivotal to develop techniques that enable monitoring and manipulation of these circuits in awake, behaving animals.

Many important technical advancements in neuroscience research with marmosets have been achieved in recent years. For example, the method of calcium imaging has been established as a promising optical alternative to monitor activity of individual neurons (Yamada et al., 2016; Kondo et al., 2018; Mehta et al., 2019). Nevertheless, extracellular single unit recordings remain the essential method in systems neuroscience due to their unparalleled temporal resolution and

37 ability to record from almost any location in the brain (Steinmetz et al., 2018). Technical

38 improvements in extracellular single unit recordings in awake marmosets were initially driven by the field of auditory research (Eliades and Wang, 2008; Remington et al., 2012; Roy and Wang, 2012). These recordings mostly utilized tungsten microelectrodes, which have limitations in terms of electrode density and geometric arrangement of recording sites. To overcome these issues,

42 silicon-based microelectrode arrays have recently been established in awake marmosets 43 (Johnston et al., 2019; Pomberger and Hage, 2019; Davis et al., 2020; Walker et al., 2021). These contributions have paved the way for better access to the neural circuits of the marmoset brain. 
47 characterization of response properties of neurons is almost entirely based on experiments performed under anesthesia. In contrast, data from visual areas in awake marmosets is still very scarce (Porada et al., 2000; Johnston et al., 2019; Davis et al., 2020). Even more strikingly, there is only one study of single unit recordings in awake marmoset primary visual cortex (V1) (Porada et al., 2000), in stark contrast to the wealth of studies on this area in other species. Hence, the relative lack of published work in awake animals emphasized the need to develop suitable recording approaches.

Technical as well as conceptual advancements have revealed that computations in the brain are carried out by populations of neurons (Saxena and Cunningham, 2019). These populations are distributed within and across areas (Poggio, 2011; Panzeri et al., 2015). Thus, it is of great interest to be able to record from both, local populations, and from distributed populations across multiple areas simultaneously. For this reason, implant designs should be compatible with modern electrode technology, such as high-density silicon probes optimized for these applications, and they should ideally allow to target multiple brain regions simultaneously (Shobe et al., 2015; Steinmetz et al., 2021).

Importantly, beyond the correlative evidence that can be obtained from neural recordings, direct manipulation of neural activity can be used to gain insight into the causal link between neural circuits and behavior (Wolff and Ölveczky, 2018). Optogenetics is a powerful tool for such questions, because it offers the necessary spatiotemporal and genetic precision (Fenno et al., 2011). The principal feasibility of optogenetic stimulation techniques in marmosets has already been demonstrated (Macdougall et al., 2016; Komatsu et al., 2017; Ebina et al., 2019). However, the integration of neural recordings, optogenetics and behavioral manipulation is still lacking. Therefore, the aim of this work was to integrate these components into a well-engineered design that enables state-of-the art experimental access in the awake, behaving marmoset. 
71 The approach presented here is based on semi-chronic recordings from multiple high-density

72 silicon probes. It makes use of a light-weight titanium chamber, fabricated with metal 3D-printing

73 technology, while surgical procedures are streamlined by means of 3D printed guides and

74 implantation holders. We demonstrate multi- and single-unit recordings from two visual areas with

75192 channels simultaneously and show that optogenetic stimulation of visual area V6 can

76 influence the animal's behavior in a perceptual detection task. Thus, we demonstrate for the first

77 time neural recordings and optogenetic stimulation in combination with behavioral manipulation

78 in the awake behaving marmoset. 
bioRxiv preprint doi: https://doi.org/10.1101/2021.10.30.466578; this version posted November 2, 2021. The copyright holder for this preprint (which was not certified by peer review) is the author/funder, who has granted bioRxiv a license to display the preprint in perpetuity. It is made available under aCC-BY 4.0 International license.

\section{Results}

a)

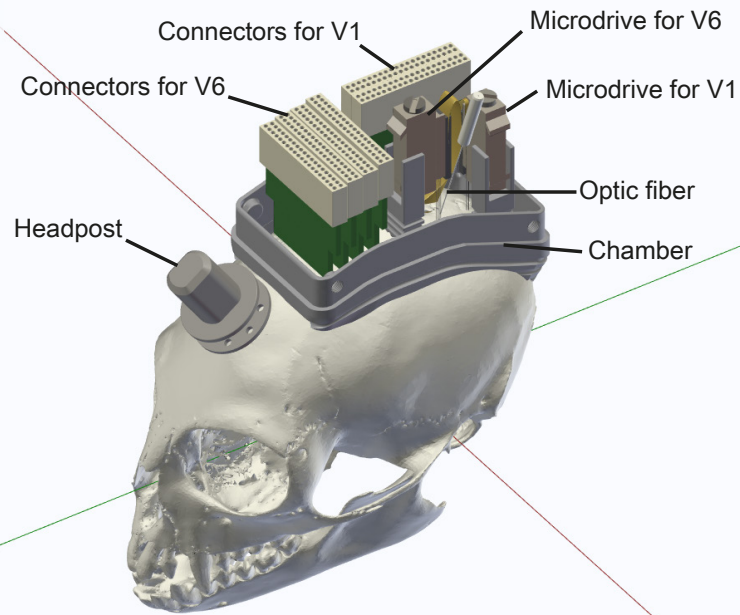

c)
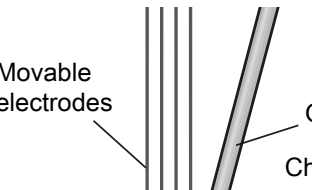

Optic fiber

Chamber wall

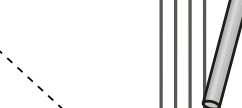
Silicone
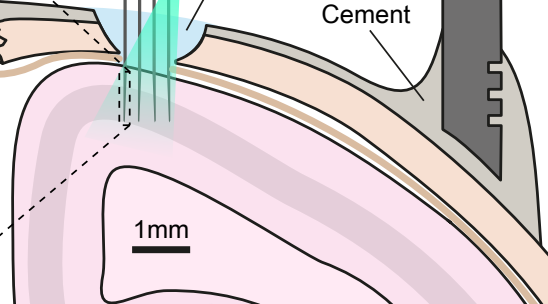

Figure 1 | Implant design and recording approach. a)
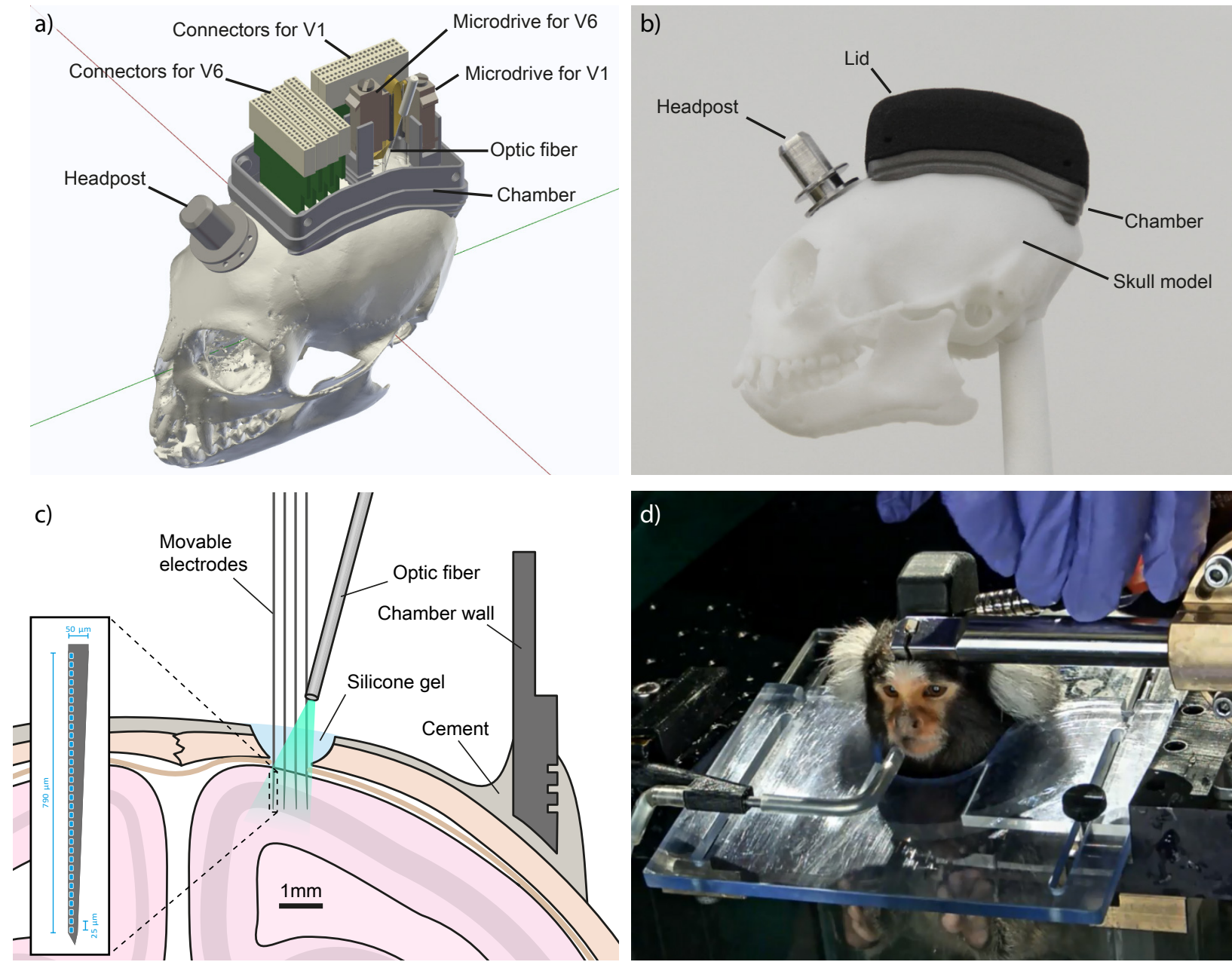

four-shank silicon probe with $4 \times 32$ channels is attached to the microdrive targeting area V6. A two-shank silicon probe with $2 \times 32$ channels is targeting area $\mathrm{V} 1$. The four connectors at the anterior end of the chamber are wired to the probe in area V6. The two connectors at the posterior right side of the chamber are routed to the probe in V1. An optic fiber (200 $\mu \mathrm{m}$ diameter) is placed above the V6 craniotomy with an external micromanipulator (not shown for clarity). The headpost for stabilizing the animal during recording is placed in front of the chamber. b) Side view photograph of a skull model with headpost, chamber and flat lid as used after implantation of headpost and chamber. c) Illustration of a coronal section of the target location in area V6. Craniotomy, electrodes and chamber are drawn to scale. Inset shows magnified view of electrode layout. d) Photograph of Monkey A while head-fixed and facing the monitor, during opening of the tall lid used after electrode implantation. The photograph shows the animal with the final 192-channel implant. 


\section{Implant design and recording approach}

92 Our goal was to design a small and lightweight implant that utilizes modern high-density silicon

93 probes while providing access to optogenetic stimulation techniques in awake behaving marmosets.

The complete implant consists of multiple parts: Headpost, chamber, microdrives, stabilizers, silicon probes and printed circuit boards (PCBs) holding the connectors (Fig. 1a). The 3D printed titanium chamber was designed to smoothly fit onto the surface of the marmoset skull (Fig. 1a, b). This was achieved by using a computed tomography (CT)-based skull model as anatomical reference for the curvature of the bottom of the chamber. The chamber houses six PCBs with connectors, which relay the neural signals from two silicon probe arrays: A four-shank $4 \times 32$ channel silicon probe is attached to a microdrive targeting visual area V6. A two-shank $2 \times 32$ channel silicon probe is located at the posterior end of the chamber to target visual area $\mathrm{V} 1$, amounting to a total of 192 channels. Both probes are implanted in the left hemisphere (Fig. 1a).

It is often advantageous to be able to move electrodes to a new recording position after signal decay, or in order to target a particular depth within the brain structure of interest. Therefore, we mounted the probes to microdrives which allow for up to $5 \mathrm{~mm}$ vertical travel. This makes it possible to change the recording position along the depth axis if required. Both microdrives are attached to titanium stabilizers that are 3D printed from the same material as the chamber. The stabilizers are intended to provide additional rigidity after implantation. Furthermore, they minimize the gap between the bottom of the microdrive and the skull, which needs to be filled with 111 cement during implantation. Thus, the stabilizers also make the implantation process easier and 112 faster.

113 Silicon probes are implanted through a small ( $\approx 2 \mathrm{~mm}$ diameter) craniotomy (Fig. 1c). After 114 superficial insertion of the probes into the brain, the craniotomy is sealed with a transparent 
silicone gel (Jackson and Muthuswamy, 2008). Optogenetic stimulation can then be performed by pointing an optic fiber at the craniotomy such that the light penetrates through the silicone into

117 the tissue (Fig. 1c). The optic fiber is held by an external micromanipulator that guarantees flexible and precise positioning.

119 To allow stabilization of the animal's head during recordings, a headpost was implanted in front of the chamber (Fig. 1a, b). Both, the headpost as well as its holder (Fig. 1d) were produced by standard CNC milling from medical-grade titanium (Ti6Al4V). 3D printing was not viable here, because it does not offer the precision necessary for the fit between headpost and its holder, without substantial post-processing (Chen et al., 2017). However, alternative headpost designs could overcome this limitation (see Discussion).

The inside of the chamber is protected by a 3D printed nylon lid that can be secured by four small screws on the side of the implant (Fig. 1a, d). Threads for the screws were manually added after 3D printing. The use of 3D printed lids makes it possible to rapidly and flexibly produce multiple versions of lids. Before electrode implantation, the inside of the chamber does not contain any parts other than the (optional) reference wires. Therefore, the initial version of the lid was flat and could later be replaced by a taller version. This procedure allowed the animals to gradually get habituated to the size and weight of the final implant. Fig. 1b, shows a photograph of the chamber on a skull model with the flat version of the lid and the headpost in place. well, without the necessity of post-implantation wound care. None of the 3D printed nylon lids did require replacement, even after several months of use with almost daily opening and closing.

136 Three of the five animals were subsequently implanted with electrodes in areas V1 and V6.

137 Figure 1d shows a photograph of the final implant in Monkey A during opening of the lid just prior 138 to electrophysiological recording. 
a)

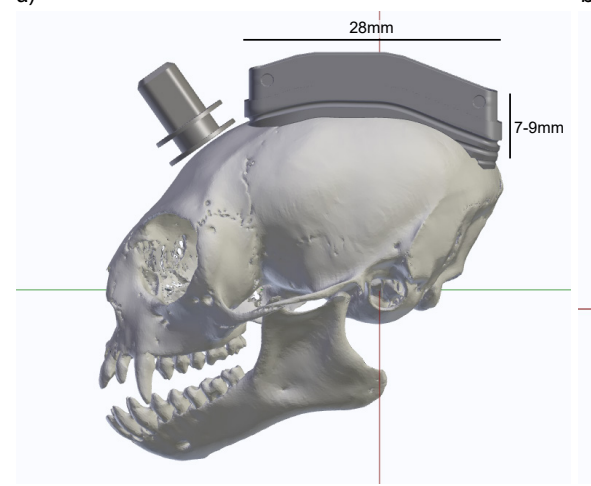

b)

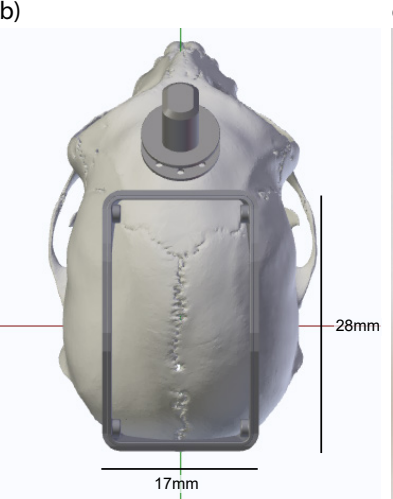

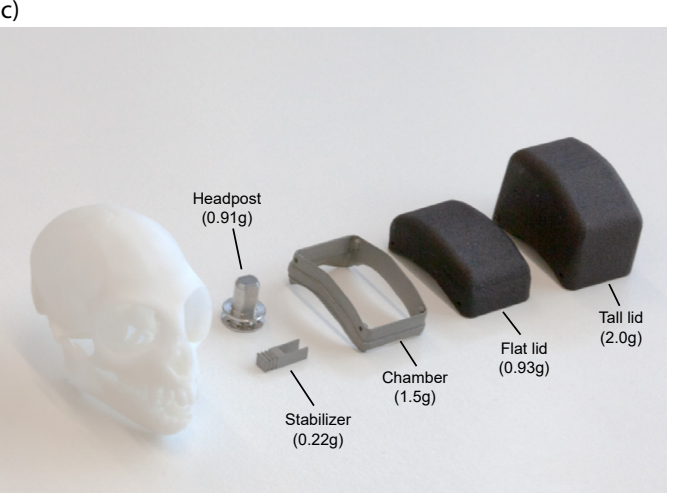

Figure 2 | Implant size and weight. 3D rendering of side view (a) and top view (b) of a marmoset skull with headpost and chamber in target position, aligned in stereotaxic coordinates. Red line indicates interaural axis. Green line indicates anterior-posterior axis. c) Photograph of the CNC machined and 3D printed parts of the implant next to a skull model. Weights are indicated in parentheses.

Size and weight minimization of an implant are important design factors when working with small animals. These factors are not only crucial in order to ensure the welfare of the animal, but also facilitate the study of natural behaviors (Kondo et al., 2018; Courellis et al., 2019).

The chamber was designed to span $28 \mathrm{~mm}$ in the anterior-posterior axis and $17 \mathrm{~mm}$ in the mediolateral axis of the skull (Fig. 2 a, b; outer chamber dimensions). We restricted the lateral extent of the chamber such its implantation required only minimal detachment of the temporal muscle from the bone. Consequently, no resection of the muscle was necessary. The sides of the chamber extended laterally only 1-2 $\mathrm{mm}$ beyond the superior temporal lines of the skull. This design allows targeting a large number of dorsal brain areas for neural recording and stimulation (Suppl. Fig. 1).

The height of the final implant depends on the selection of electrodes and connectors inside the chamber. The chamber itself (without lid) protrudes only 7-9 $\mathrm{mm}$ from the surface of the skull. When closed with the flat lid (e.g. without probes installed), it reaches a height of 12-14 mm from the skull. After implantation with silicon probes and connector PCBs as used here, the chamber is closed with a taller version of the lid, and the implant reaches a height of $20-22 \mathrm{~mm}$ from the skull. 
The total weight of the implant depends on its size and the density of the materials that are used.

Recent advancements in metal 3D printing make it possible to accurately produce complex shapes from medical-grade titanium (Ti6Al4V). The mechanical strength of titanium allowed us to

162 reduce the wall thickness of the chamber to $0.5-1 \mathrm{~mm}$ (Fig. 1c and Fig. 2b), which resulted in a 163 weight of only $1.5 \mathrm{~g}$ for the chamber (Fig. 2c). Headpost and stabilizers had a weight of $0.91 \mathrm{~g}$ 164 and $0.22 \mathrm{~g}$, respectively. Lids were produced from a polyamide (PA12 nylon). Polyamides such 165 as nylon show exceptional tensile strength, resistance to abrasion and can be 3D printed in a cost-effective way (O'Connor et al., 2018). Weights of the lids for the flat and tall version were $0.93 \mathrm{~g}$ and $2.0 \mathrm{~g}$, respectively. Thus, the total resulting weight of the implant was approximately only $8 \mathrm{~g}$, including headpost, chamber, silicon probes, microdrives, stabilizers, connectors and cement.

The implant design presented in this work combines several significant improvements over

171 existing methods. It is small and extremely lightweight and enables recordings with a large 172 number of channels as well as access for optogenetic stimulation. Because most parts are 3D 173 printed, they can be manufactured very quickly at low cost and can be rapidly adapted for other 174 methods such as calcium imaging or functional ultrasound imaging.

\section{Two-stage implantation procedure}

176 Surgeries for experiments of the type described here often include a number of critical steps, such

177 as: precise alignment of several independent parts, insertion of electrodes in multiple target areas 178 and injection of viral vectors. Performing any of these steps is challenging even individually, and 179 combining all of them in one surgery increases the risk of failure. To maximize chances of surgical success, we adopted a two-stage implantation procedure and made use of customized 3D printed 
182 (Surgery 1). After appropriate recovery time, a second surgery was performed, in which a viral 183 vector was injected and several silicon probes were implanted (Surgery 2).

\section{Surgery 1: Implantation of chamber and headpost}

185 At the beginning of the first surgery, the animal was placed in a stereotaxic apparatus, and the 186 skull was prepared for the implant (see Materials and Methods). Chamber and headpost could 187 then be lowered onto the skull surface for alignment. Precise alignment of the chamber relative to the skull was crucial, because it ensured that the chamber could later be used as positional reference for the stereotaxic coordinate system. Both, chamber and headpost were held by a custom implantation holder that was attached to a micromanipulator (Fig. 3a). Prior to the surgery, cross-shaped markers on the sides of the holder were used for alignment to the interaural line (i.e. the axis of the ear bars). This assured correct positioning of the chamber in the anteriorposterior axis. During the surgery, a downward-pointing wedge integrated into the holder was aligned to the central skull suture, to assure correct positioning in the medio-lateral axis removed to allow better access for the subsequent surgical steps.

Marmosets have thin skulls and a narrow subdural space, which can make the use of bone screws problematic. Therefore, we used only dental adhesive and cement to secure the implant to the skull (Johnston et al., 2018). To this end, the skull surface was cleaned, roughened with a metal brush and coated with dental adhesive before a thin layer of cement was applied. Two platinum

201 wires were implanted epidurally anterior to the chamber, serving as backup reference wires. Next, the implantation holder was returned to the previously determined antero-posterior and mediolateral position, and lowered until the chamber contacted the skull. Following a final visual inspection of alignment, the headpost and chamber were cemented in place (Fig. 3b). After the 
of the surgery, the flat version of the 3D printed nylon lid was used to close the chamber (Fig. 3d).

207 The animal was then allowed to recover for two weeks and subsequently underwent head-fixation

208 training.

209 Variability in head morphology between animals can lead to inaccuracies during stereotaxic

210 surgeries. Therefore, after the first surgery, we obtained anatomical data of the skull and implant

211 via computed tomography (CT) scans (Fig. 3e-g). Appropriate thresholding of the CT images

212 allowed segmentation of the bone (shown in transparent gray), and of metal and radio-opaque

213 cement (shown in green). The cement layer in the center of the chamber was very thin and is

214 therefore not visible everywhere in the segmented data, even though the skull inside the chamber

215 was completely covered with cement. Also, note that the platinum wires appear thicker than they

216 actually are due to the high CT contrast of the metal.

217 After segmentation, the inside of the animal-specific skull model was used to fit an MRI-based

218 template marmoset brain (Liu et al., 2018). This approach can be justified under the assumption

219 that the gap between bone and the brain is very small. Fits were performed manually by

220 translating and scaling in all three spatial dimensions, and rotating in the pitch axis. The resulting

221 fit of the template brain and its area delineations can then serve as individualized anatomical

222 reference for each animal. Thereby, we obtained the precise positions of our target areas in the

223 same reference frame as the chamber visible in the CT. Note that this CT-based targeting

224 refinement was only used in marmosets $\mathrm{D}$ and $\mathrm{U}$. 

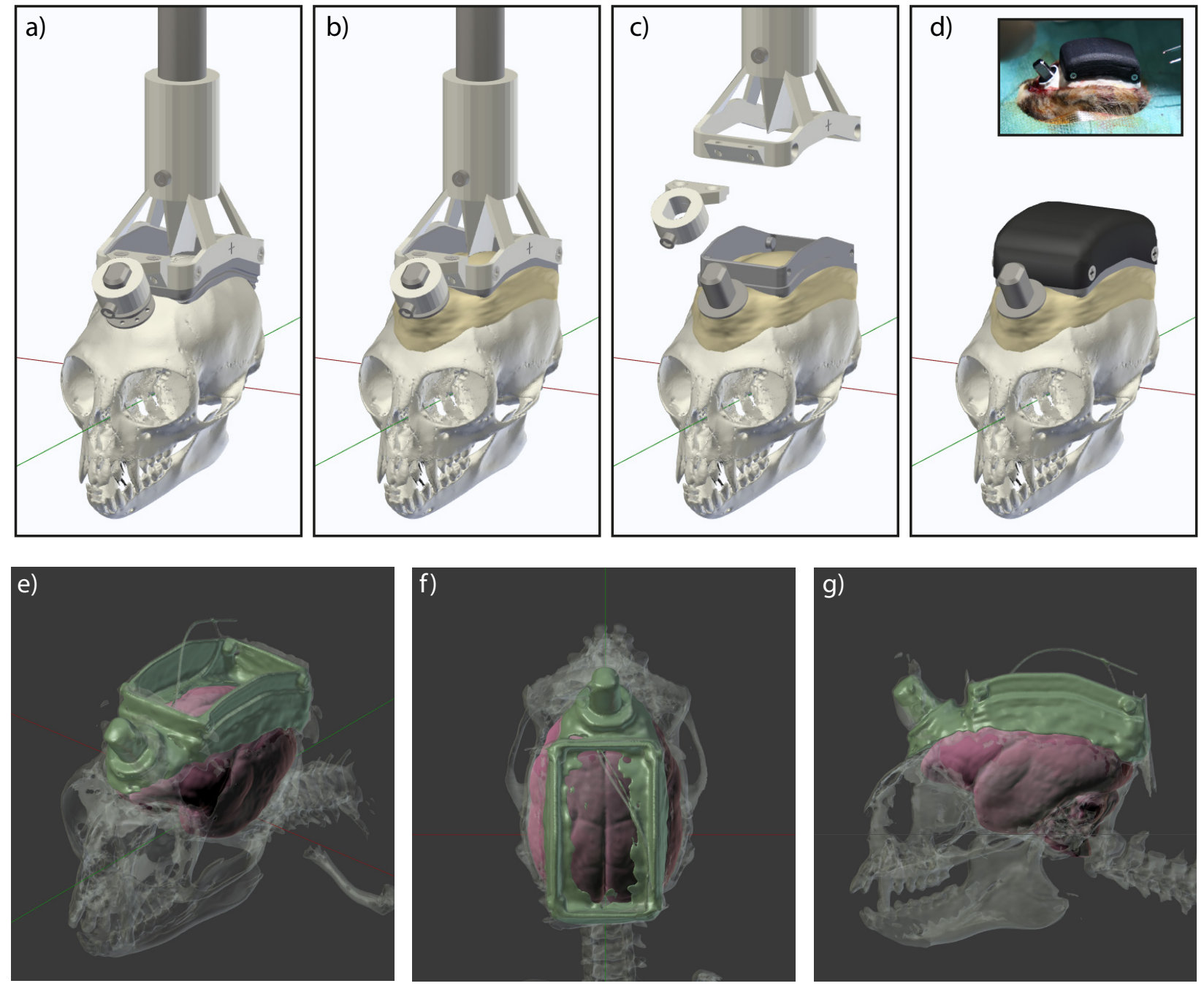

Figure 3 | Surgery 1: Implantation of chamber and headpost. a) Chamber and headpost were held by a custom implantation holder that was attached to a micromanipulator. Note the cross-shaped markers on the side of the holder, used for alignment to the interaural axis, prior to the surgery. A wedge-shaped guide pointing downwards in the center of the holder was used for medio-lateral alignment to the central skull suture. b) Following skull preparation, the aligned chamber and headpost were cemented onto the skull. c) Once the cement had hardened, chamber and headpost were released from the holder. d) The chamber was closed with a 3D printed nylon lid for protection. Inset shows photograph of the implant at the end of the first surgery. e) Near-isometric projection, f) top view and g) side view of the 3D segmentation from a CT scan after the first surgery in monkey D. Radio-opaque cement, metal parts and reference wires show the highest contrast and are colored in green. Bone is shown in semi-transparent gray. The fitted MRIbased template brain is shown in red. 


\section{Surgery 2: Injection of the viral vector and implantation of silicon probes}

237 To assure correct positioning in the second surgery, the implantation holder from the first surgery

238 (Fig. 3a-c) was used to re-align the animal's head via the previously implanted chamber: After ensuring sufficient depth of anesthesia, the lid was removed, and the chamber attached to the animals' skull was re-inserted into the holder. This effectively re-aligned the skull of the animal to precise stereotaxic coordinates as defined by the holder and the chamber. Subsequently, a highprecision articulated arm was used to fix the animals' head position via the implanted headpost. After locking the articulated arm, the chamber holder was removed. Thus, the use of ear bars and animal.

Next, the inside of the chamber was disinfected with $\mathrm{H}_{2} \mathrm{O}_{2}$ and ethanol. A 3D printed guide was temporarily placed on the chamber and used to mark the target positions for the craniotomies over areas V1 and V6 of the left hemisphere (Supplementary Fig. 2). In Monkey A, coordinates for the guide were based on Paxinos et al., 2012, in monkeys $D$ and $U$, coordinates were based on area delineations of Liu et al., 2018 after CT-based fitting to the individual animal, as described

251 above.

252 Two platinum wires, serving as reference electrodes, were then implanted subdurally at the 253 anterior end inside the chamber, through a small burr hole ( $\approx 2 \mathrm{~mm}$ diameter). Next, two small burr 254 holes were made at the target locations for the electrodes over V1 and V6. A durotomy of 255 approximately $1.5 \mathrm{~mm}$ was performed over area V6, and the viral vector was injected (Fig. 4a). 256 After a short waiting time for diffusion of the vector into the tissue, the needle was slowly 257 retracted.

258 A custom 3D printed implantation holder was then lowered into the chamber (Fig. 4b). The holder 259 was prepared prior to the surgery to hold all necessary components for the implantation: two 
microdrives (with silicon probes and stabilizers attached) and six connector PCBs. The three main components (connector PCBs, V1 microdrive with probes and V6 microdrive with probes) were held by separate parts of the implantation holder, enabling independent movement in the z-axis.

263 This independence allowed sequential implantation of the components. To this end, the holder 264 was initially prepared such that the connector PCBs were at the lowest position and were thus 265 implanted first (Fig. 4b). Connector PCBs were positioned via the micromanipulator just above 266 the cement layer on the skull, and were then cemented in place. After curing, the part of the 267 implantation holder securing the connector PCBs was removed (Fig. 4c). This resulted in better 268 visibility and allowed for independent movement of the microdrives holding the silicon probes (Fig. 269 4c). Next, the probe array for area V6 was implanted. In order to insert the silicon probe into the 270 cortex at the optimal position relative to the durotomy and the local cortical vasculature, the 271 anterio-posterior and medio-lateral positions of the implantation holder were fine-tuned before 272 probe insertion. After the probe was slowly inserted into the superficial part of the cortex $273(<500 \mu \mathrm{m})$, the microdrive with its attached stabilizer were cemented into the chamber. 274 Subsequently, the part of the implantation holder that was securing the V6 microdrive was 275 removed, too. The same procedure was performed for area V1, and the implantation holder was 276 completely removed (Fig. 4d). Both craniotomies were then sealed with soft silicone gel (Fig. 1c).

277 Animals recovered very quickly after the second surgery and were brought into the recording 278 setup within a few days. To visually inspect the position of the microdrives and PCBs, we obtained 279 a CT scan from Monkey A after the second surgery (Figures $4 \mathrm{e}-\mathrm{g}$ ). The high contrast metal parts 280 of the connectors and microdrives with stabilizers are visible in green color. Bone is shown in 281 semi-transparent gray and the fitted MRI-based template brain in red. 

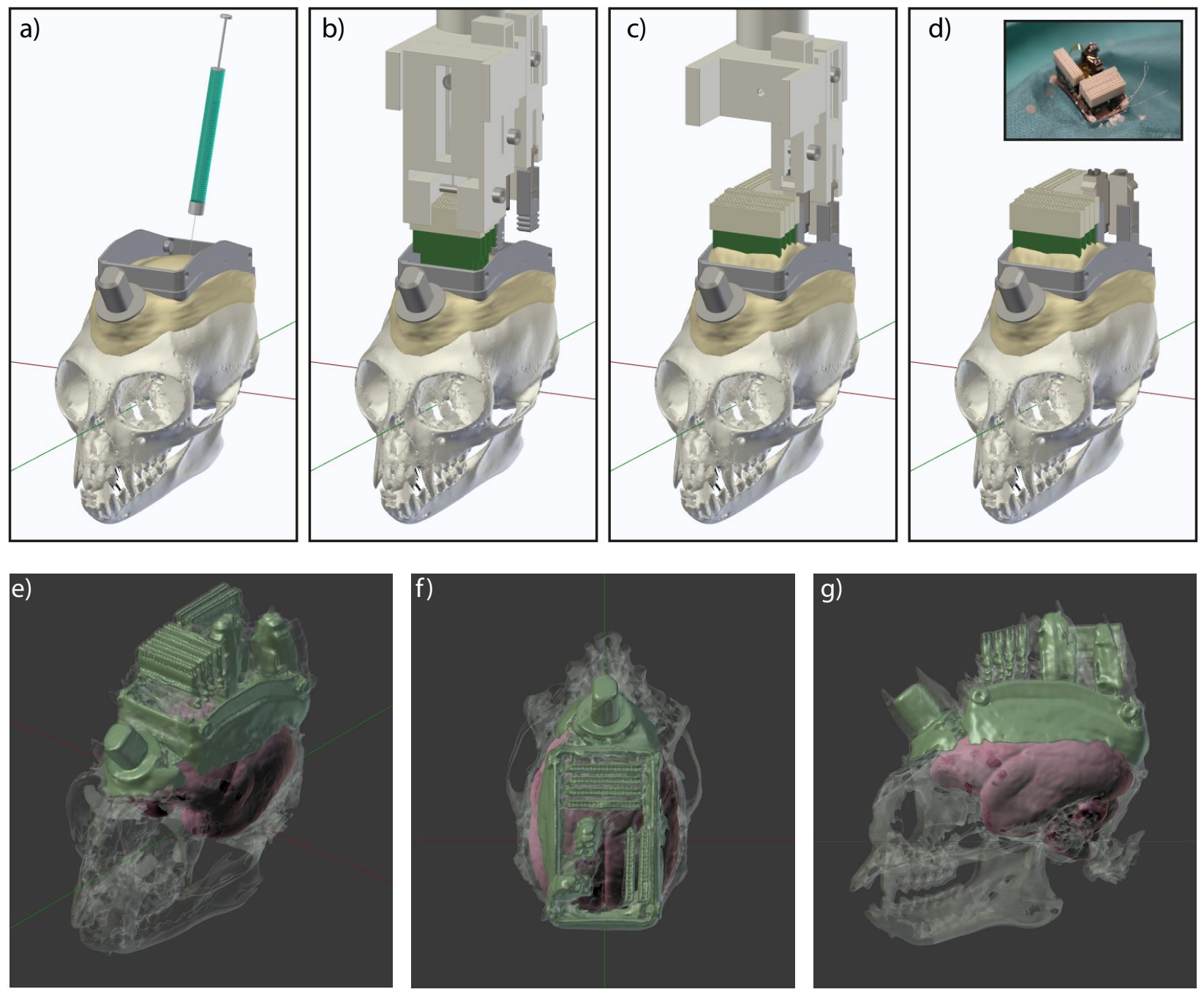

Figure 4 | Surgery 2: Injection of the viral vector and implantation of silicon probes. a) After stereotaxic alignment of the skull via the implantation holder and the chamber, a viral vector was injected into area V6. b) A custom implantation holder, carrying connector PCBs, electrodes and microdrives was lowered into the chamber. c) First, the connector PCBs were cemented in place and the respective part of the holder was removed to ensure better access and visibility. Electrodes were then lowered sequentially into the two brain areas, and the respective microdrives were cemented into position. d) After all parts were secured, the holder was completely removed. Inset shows photograph at the end of the second surgery. e) Near-isometric projection, f) top view and g) side view of the 3D segmentation form a CT scan after the second surgery in Monkey A. Radio-opaque cement and metal parts (including connectors and microdrives) show the highest contrast and are colored in green. Bone is shown in semi-transparent gray. The fitted MRI-based template brain is shown in red. 


\section{Simultaneous recording in areas V1 and V6}

294 After slowly lowering the probes into the brain, clear spiking activity was visible across several 295 recording sites in areas V1 and V6 (Fig 5a).

In order to test visual responsiveness and spatial selectivity, we performed receptive field (RF) mapping with multi-unit-activity (MUA). Flashing annulus and wedge stimuli were presented while the animal was maintaining its gaze on a central fixation point. Reverse correlation analysis was used to locate RF centers across the whole monitor. A detailed account of the RF mapping procedure can be found in Jendritza et al., 2021. As expected from the implantation target position, RFs in area V1 were located in the lower right visual field (Fig. 5b). Furthermore, RFs showed substantial overlap for all electrodes along a given probe shank (Fig. 5b, black outlines at bottom).

Next, we presented static square-wave gratings to the animals. MUA following visual stimulation with gratings was visible across several recording sites and peaked shortly after stimulus onset the following criteria: (1) The absolute magnitude of trial-averaged MUA exceeded the value of 3 STDs over the baseline (|z-score|>3) and (2) the distribution of MUA values were significantly

(Fig. 5f, g; $n=27$ out of 32 sites). 
V
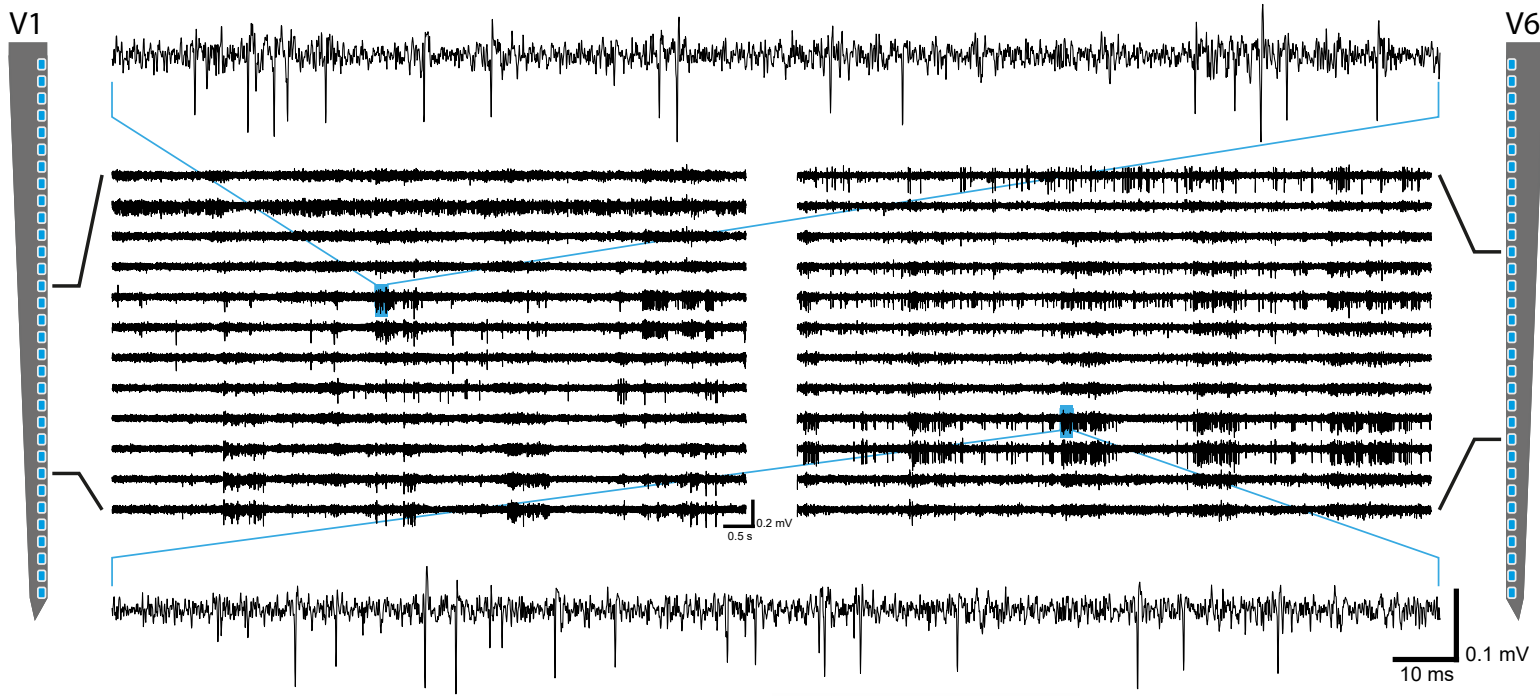

b)

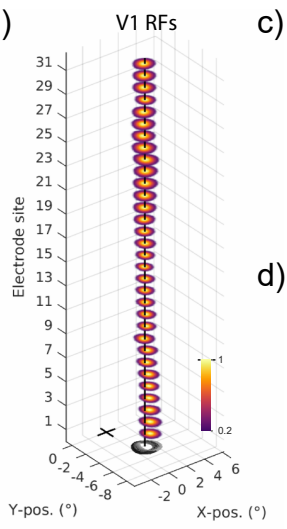

c)
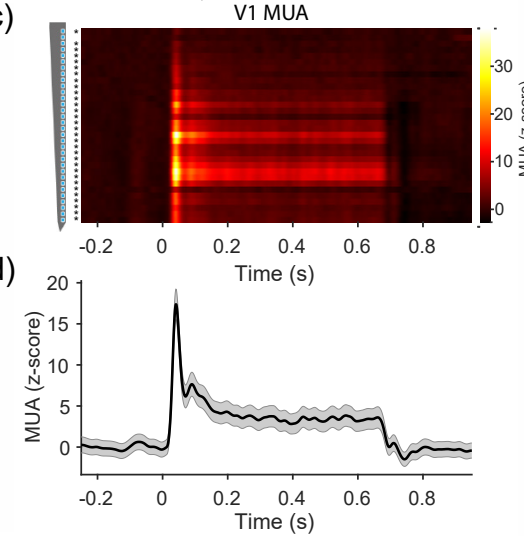

e)

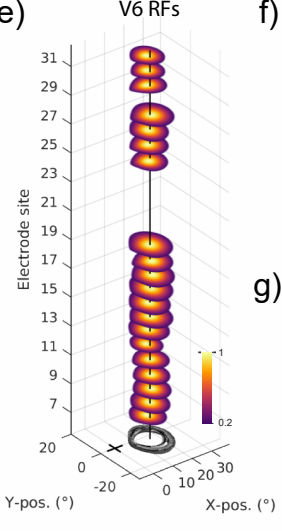

)

g)
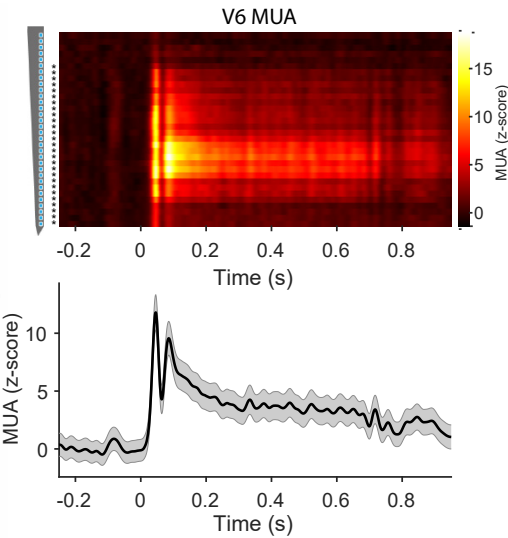

Figure 5 | Neural recordings in areas V1 and V6. a) Band-pass filtered signal (0.3-6 kHz) from example recording sites across one shank in area V1 (left) and area V6 (right). Top and bottom traces show magnified view of the respective example signals in V1 and V6. b) Receptive field (RF) locations calculated from the normalized multi-unitactivity (MUA) of all significantly modulated sites along the example shank ( $n=32$ out of 32 sites). Outlines of RFs are shown at the bottom in black to gray lines from most superficial to the deepest channel. The vertical black line indicates the median RF location across all sites. The black cross marks the position of the fixation point at the center of the monitor. c) Trial-averaged MUA along the example shank around the time of visual stimulation with gratings. Asterisks on the left indicate significant modulation between pre-stimulation baseline $(-0.25$ to $0 \mathrm{~s})$ and post-stimulus time ( 0 to

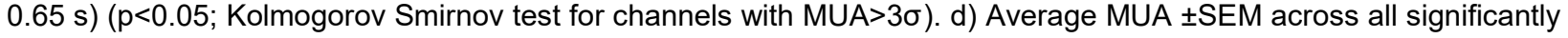
modulated sites from the example V1 shank ( $n=31$ out of 32 sites). e-g) Same as b-d but for example shank in area V6 ( $n=20$ out of 32 sites were modulated during RF mapping; $n=27$ out of 32 sites were modulated during visual 327 stimulation with gratings). MUA was smoothed with a Gaussian window ( $\sigma=8 \mathrm{~ms})$. Note different axis scaling between 328 panel $b$ and e. Data for RF mapping and visual stimulation with gratings were recorded in separate sessions in 329 Monkey A. 


\section{Single unit responses}

331 Having established the overall responsiveness and visual selectivity of MUA, we next sorted 332 spiking data into single units. Spike sorting was performed semi-automatically with the "Kilosort" 333 algorithm (Pachitariu et al., 2016). Figure 6 depicts, in the left panel of each column, the average waveform across all 32 channels of the relevant electrode shank. Due to the fine inter-electrode spacing $(25 \mu \mathrm{m})$, spike waveforms of each identified neuron were detectable as a spatial (and temporal) pattern across multiple sites. Raster plots and corresponding peristimulus time histograms (PSTHs) around the time of visual stimulation (black bar on top, $0.65 \mathrm{~s}$ duration) can be seen in the first and second row of Figure 6. The inset in the second row shows orientation tuning curves calculated from the average spiking activity during the stimulus period (0-0.65 s). Peak-normalized auto-correlograms for all spikes during the recording session are shown in the third row. The bottom row shows each unit's firing rate over the course of a recording session, documenting that all units were stable throughout the session.

343 The observed single units exhibited different response characteristics, as expected from neural recordings in visual cortex. Examples in Figure 6 were selected in order to depict the variety of response profiles present in the data. The units in Fig. $6 a$ and $b$ were recorded in area V1. Unit a) was strongly visually driven, showed a sharp peak after stimulus onset and exhibited clear orientation tuning, reminiscent of the principal cells in V1 of the anesthetized marmoset (Yu and Rosa, 2014). The unit in Fig. 6b was suppressed during the time of visual stimulation, had a relatively high baseline firing rate, and was orientation tuned. Unit c) and d) are examples recorded in area V6. Unit c) showed a sustained activation and orientation tuning, similar to

351 previous reports in V6 (Lui et al., 2006). In contrast, unit d) responded only transiently and 352 exhibited only weak orientation tuning, potentially due to a non-optimal spatial frequency of the 353 visual stimulus. 

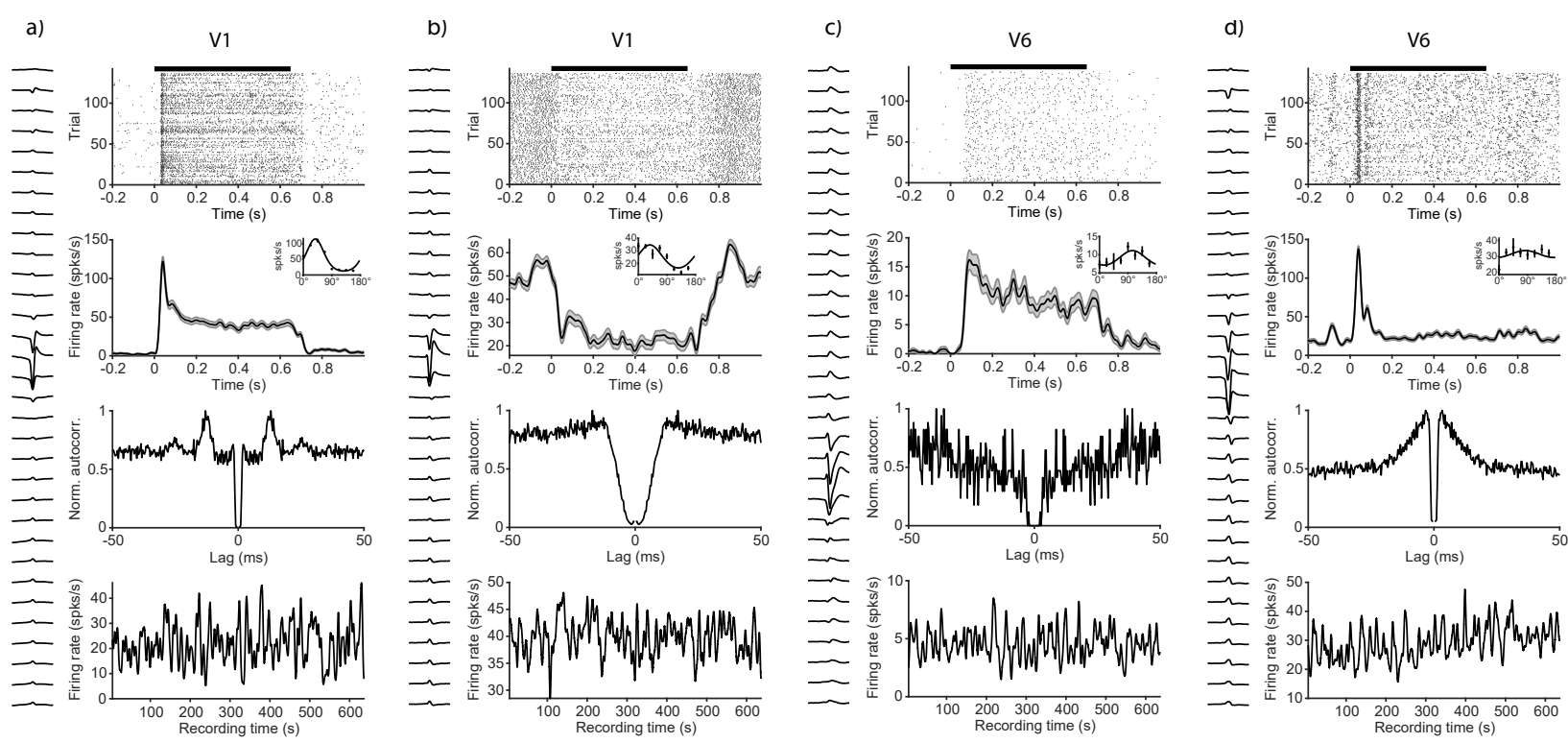

Figure 6 | Single unit examples from areas V1 and V6. Examples of four visually modulated single units (a-d). The left side of each column shows the mean waveform across all 32 recordings sites of the electrode shank on which the largest absolute amplitude was detected. Top row: Spiking raster plot around the time of visual stimulation. Black bar on top indicates stimulus duration $(0.65 \mathrm{~s})$. Second row: trial averaged and smoothed (Gaussian window, $\sigma=10 \mathrm{~ms}$ ) peristimulus time histogram (PSTH). Inset shows orientation tuning curves calculated from the mean activity during the stimulus period $(0-0.65 \mathrm{~s})$. Error bars and shaded area indicate SEM. Third row: peak-normalized auto-correlogram for all spikes across the recording. Bottom row: Smoothed firing rate (Gaussian window, $\sigma=2 \mathrm{~s}$ ) across the entire session, indicating stability of the recordings. All examples from one recording session in Monkey A.

\section{Optogenetic stimulation of area V6}

364 Optogenetics has become an essential tool in systems neuroscience (Deisseroth, 2015). To demonstrate that our recording approach is compatible with optogenetic stimulation techniques, we injected an adeno-associated viral vector (AAV), expressing the fast channelrhodopsin variant 'Chronos' (Klapoetke et al., 2014) under control of the CamKIla promotor into area V6. Expression under the CamKIla promotor is almost exclusively restricted to excitatory neurons (Gerits et al., 
371 (Fig. 1c). The optic fiber was coupled to a laser that could be directly modulated with arbitrary 372 waveforms. Stimulation was performed with sinusoidal waveforms at a peak amplitude of $25 \mathrm{~mW}$.

373 One example trial in which stimulation was performed with an $80 \mathrm{~Hz}$ sinusoidal waveform is 374 depicted in Fig. 7a. Optogenetically-induced spiking was visible across several channels. 375 Analysis of the trial-averaged MUA revealed clear optogenetic activation time-locked to the laser 376 waveform, for all 32 channels along the example shank (Fig. 7b). The z-scored MUA averaged 377 across all trials and all modulated channels is presented in Fig $7 c(p<0.05$, Kolmogorov Smirnov

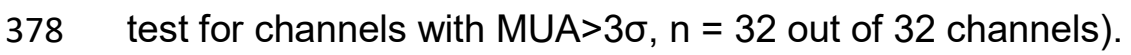

379 In order to exclude potential contamination from light-induced artifacts, we took several 380 precautions and applied appropriate controls: First, the silicon probes used in this study are 381 relatively robust against light artifacts (Chen et al., 2021). Furthermore, we avoided fast transients 382 in light intensity by stimulating with low-frequency sine waves that do not contain energy in the 383 spike frequency range. Data for MUA and SUA analysis in which optogenetic stimulation was 384 performed, were high-pass filtered with a sharp frequency cutoff (Chebyshev Type II filter) and 385 strong stop-band attenuation $(200 \mathrm{~dB})$ to remove any potential contamination from the low 386 frequency laser signal (Wu et al., 2015). Additionally, we included a control condition, in which 387 light with a wavelength of $594 \mathrm{~nm}$ with matched output power was used for optical stimulation. 388 The opsin variant used in this study should not be activated by this wavelength (Klapoetke et al., 389 2014). These controls ruled out that the observed neural activation was caused by light artifacts or other non-specific effects such as heating. 
a)

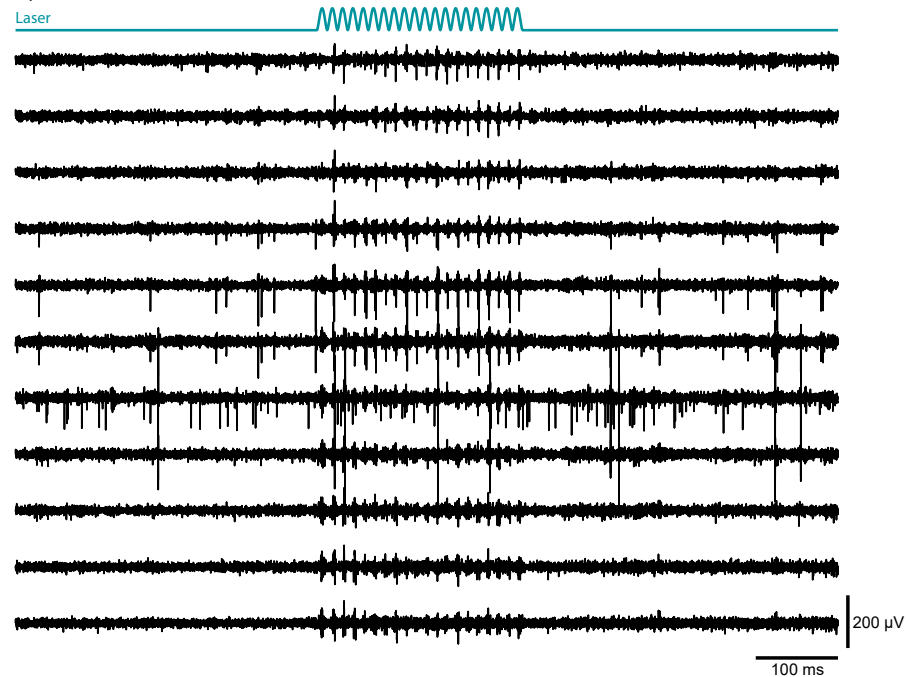

b)
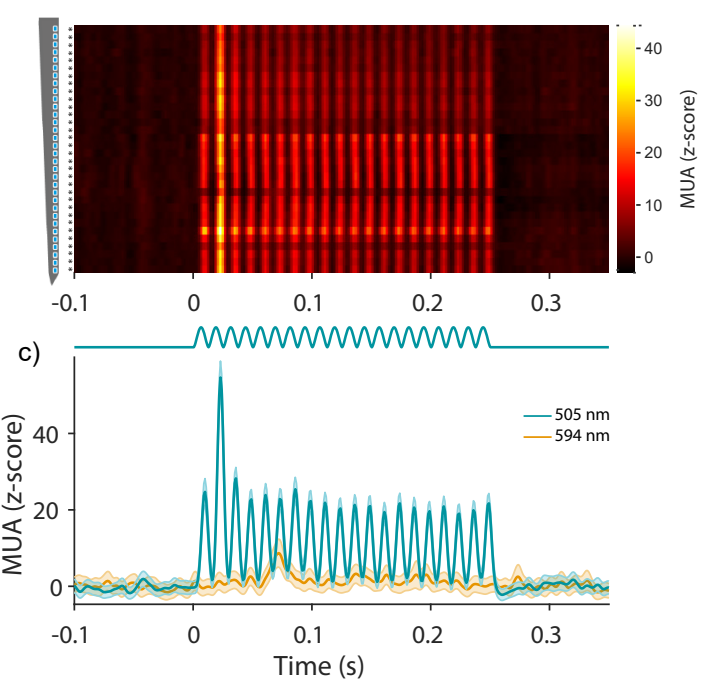

d)
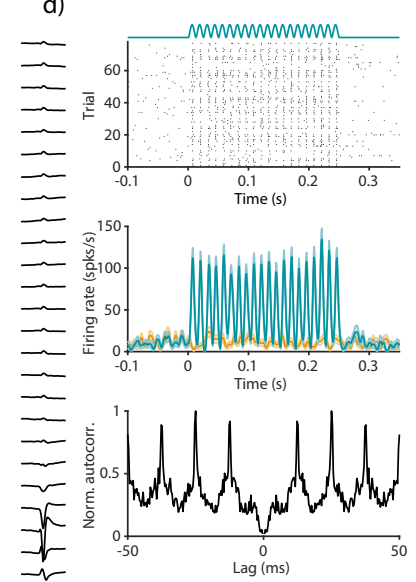

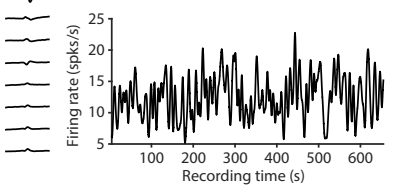

e)

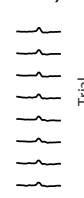

$\approx$
$\approx$
$\approx$
$\approx$
$\approx$
$\approx$
$\approx$

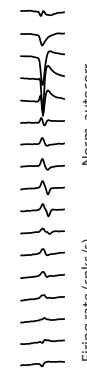

(2.
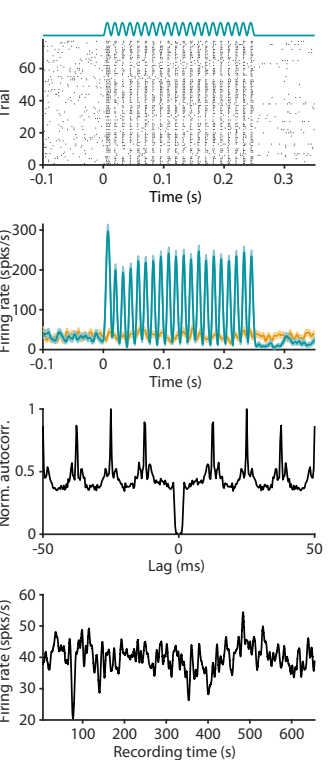

f)
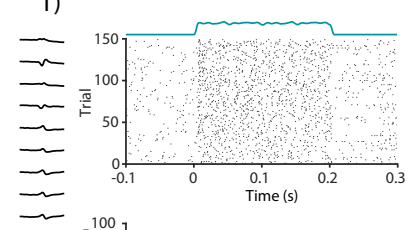

g)

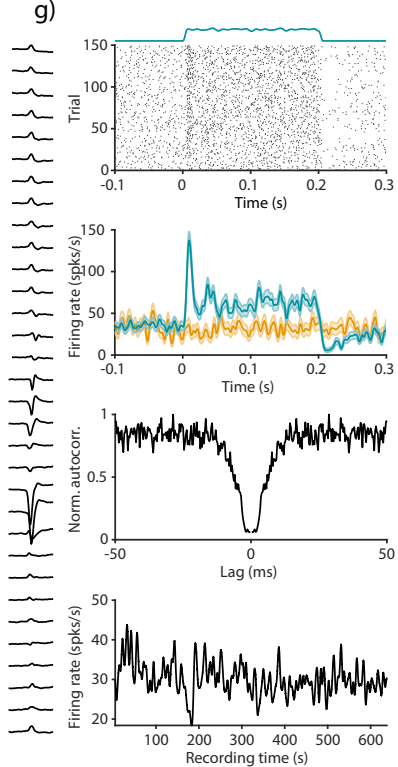

Figure 7 | Optogenetic activation of neurons in the awake marmoset. a) Example traces of band-pass filtered data during optogenetic stimulation with an $80 \mathrm{~Hz}$ sinusoidal pattern of $250 \mathrm{~ms}$ duration (25 mW peak). b) Trial averaged, zscored MUA of all recordings sites from an example shank for the $505 \mathrm{~nm}$ stimulation condition. Asterisks on the left indicate significant modulation between pre-stimulation baseline ( -0.25 to $0 \mathrm{~s})$ and stimulus time $(0$ to $0.25 \mathrm{~s})$ across all

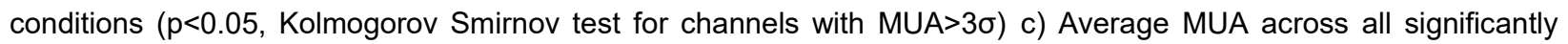
modulated channels ( $n=32$ out of 32 channels), for stimulation with $505 \mathrm{~nm}$ and $594 \mathrm{~nm}$, respectively as indicated by the color legend. $d-g$ ) Four examples of optogenetically modulated single units: d) and e) from Monkey A, f) and g) from Monkey D. The left side of each column shows the mean waveform across all 32 recordings sites of the relevant electrode shank. Top row: raster plot of spikes around the time of stimulation with $505 \mathrm{~nm}$. Average laser waveform across all trials is shown on top. Second row: trial averaged and smoothed (Gaussian window, $\sigma=2$ ms) peristimulus 
time histogram (PSTH) for stimulation conditions with $505 \mathrm{~nm}$ and $594 \mathrm{~nm}$ (same color code as in c). Third row: peakinduced rhythmicity in the autocorrelations of neurons in d) and e). Bottom row: Smoothed firing rate (Gaussian window, $\sigma=2 \mathrm{~s})$ across the entire session, indicating stability of the recordings.

Next, we spike sorted the data as described earlier in order to identify optogenetically modulated

407 single units. Four example units are depicted in Figure $7 \mathrm{~d}-\mathrm{g}$ (figure conventions are as in Figure

408 6). Figures 7d and e show examples from Monkey $A$, in which optogenetic stimulation was performed with an $80 \mathrm{~Hz}$ sinusoidal pattern. On each trial, sinusoidal waveforms started smoothly at the trough from an intensity of $0 \mathrm{~mW}$ with a peak amplitude of $25 \mathrm{~mW}$. Single unit spikes were precisely time locked to the laser stimulation (Fig 7d, e). Consistent with the trial-averaged optogenetic responses, the resulting autocorrelation analysis of SUA showed a prominent peak

413 at the reciprocal of the stimulation frequency $(1 / 80 \mathrm{~Hz}=12.5 \mathrm{~ms})$. Figure $7 \mathrm{f}$ and $\mathrm{g}$ are additional 414 examples from Monkey D, in which optogenetic stimulation was performed with sine waves of 415 different frequencies $(0,10,20,30,40,50,60,70,80 \mathrm{~Hz})$ and randomized phases. To avoid artifacts from sharp transients in light intensity, onset and offset of the stimulation waveform were

417 smoothed (see Materials and Methods for details). The resulting average laser intensity across 418 all trials is shown on top of the raster plot. In both monkeys, spiking activity of single units was 419 not affected by the control stimulation (yellow trace in second row, $594 \mathrm{~nm}$ ), and the rates 420 remained relatively stable throughout the recording session (Fig.7, lowermost row).

421 These results show that we successfully combined semi-chronic recordings in two areas with 422 optogenetic stimulation of neurons in the visual cortex of the awake marmoset. 
a)

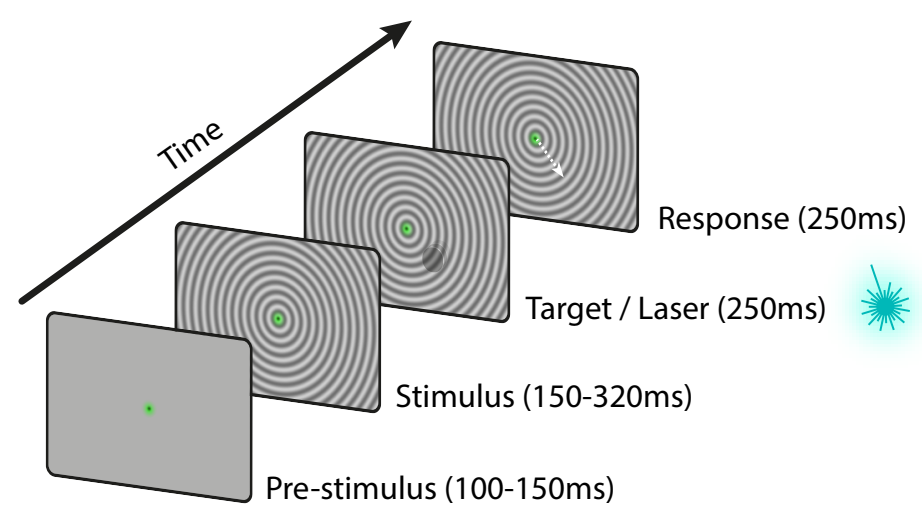

b)

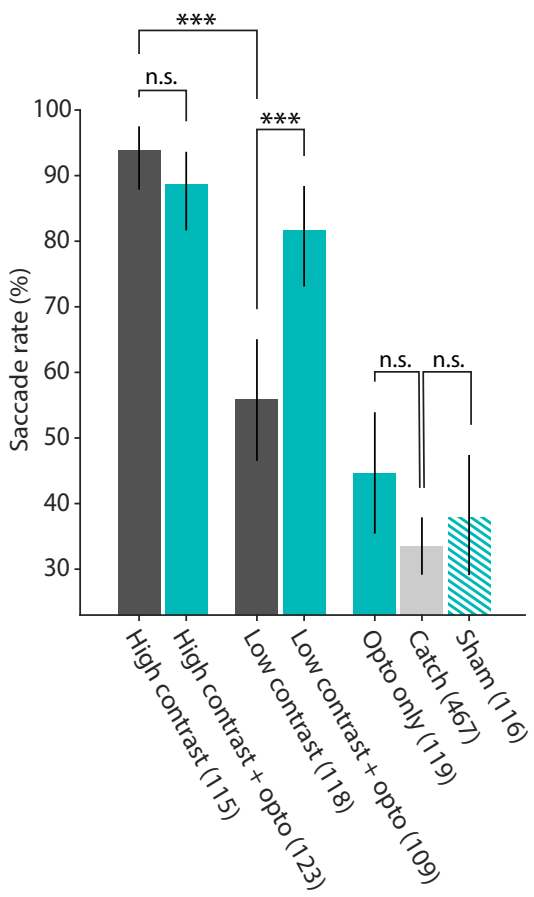

Figure 8 | Visual and optogenetic detection task and behavioral results. a) Schematic illustration of the detection task. After a brief pre-stimulus fixation period, a background stimulus was shown, followed by the onset of a small visual target with either high or low contrast. On $50 \%$ of these trials, the visual target was paired with optogenetic stimulation. Additionally, trials without visual target were included, either with effective laser stimulation ('Opto only' condition) or with control laser stimulation, in which the optic fiber was placed outside the craniotomy ('Sham' condition), or with no laser stimulation ('Catch'). All trial conditions except catch trials had identical timing and were rewarded if the monkey executed a saccade 50-500 ms after target or laser onset. b) Saccade rates for all task conditions. The animal showed increased detection performance (higher saccade rate) for high-contrast visual targets compared to low contrast targets (93.9\% vs. $55.9 \%$, Chi-squared test; $p=3.64 \mathrm{e}-10)$. Pairing high-contrast visual targets with optogenetic stimulation did not result in a difference in saccade rate (Chi-squared test; $p=0.283$ ). Saccade rate increased significantly when low contrast targets were paired with optogenetic stimulation ( $55.9 \%$ vs. $81.7 \%$; Chi-squared test; $p=1.47 \mathrm{e}-04)$. Optogenetic stimulation alone was not sufficient to be detected by the animal when compared to the false alarm rate $(44.5 \%$ vs.

$43633.4 \%$; Chi-squared test; $p=0.0521$ ). The saccade rate in the sham stimulation control condition (laser fiber positioned 4372 mm outside the craniotomy) was not different from the false alarm rate ( $37.9 \%$ vs. $33.4 \%$; Chi-squared test; $p=0.419)$. Number of trials are shown in parenthesis. Error bars indicate $95 \%$ confidence intervals. 
439 Behavioral report of optogenetic stimulation

440 In order to test whether activation of excitatory neurons in area V6 could be behaviorally reported,

441 we trained one animal (Monkey A) in a visual and optogenetic detection task (Fig.8a). The animal

442 was required to briefly maintain fixation (100-150 ms) on a central fixation point. After this period,

443 a background stimulus (full screen circular grating) was presented. After an additional 150-320

$444 \mathrm{~ms}$, a moving visual target with either low or high contrast was presented for $250 \mathrm{~ms}$. Half of these

445 trials were randomly paired with optogenetic stimulation (250 ms square pulse, $25 \mathrm{~mW}$ amplitude,

446 same onset time as visual stimulus, see Materials and Methods for details). An additional

447 condition was included in which optogenetic stimulation was performed in the absence of a visual

448 target. The monkey was rewarded for making a saccade away from the fixation point within 500

$449 \mathrm{~ms}$ from the onset time of visual and/or optogenetic stimulation. To prevent false alarms, $40 \%$ of

450 all trials were 'catch trials', in which neither an optogenetic nor a visual target appeared. In these

451 trials, the monkey was rewarded for maintaining fixation until the end of the trial. As control, we

452 randomly interleaved trials with a sham stimulation condition. Sham stimulation was identical to

453 real optogenetic stimulation (without a visual target), but the laser output was switched to a second

454 optic fiber that was placed $2 \mathrm{~mm}$ outside the craniotomy. Importantly, sham trials were rewarded

455 identical to real trials, such that the monkey would be able to benefit from any cues unspecific to

456 the optogenetic stimulation.

457 High-contrast visual targets were correctly reported in $93.9 \%$ of trials, compared to only $55.9 \%$ in

458 the low-contrast target condition (Chi-squared test; $p=3.64 \mathrm{e}-10 ; n=115$ high-contrast and $n=123$

459 low contrast trials). Pairing the high-contrast visual target with optogenetic stimulation did not

460 significantly affect detection performance (Chi-squared test; $p=0.283 ; n=115$ high-contrast and

$461 \mathrm{n}=123$ high-contrast + opto trials). A different pattern was observed for low-contrast visual targets:

462 Pairing the visual stimulus with optogenetic stimulation caused performance to improve from

$46355.9 \%$ to $81.7 \%$ (Chi-squared test; $p=1.47 e-04 ; n=118$ low-contrast and $n=109$ low- 
464 contrast + opto trials). The observed increase in saccade rate indicates that the monkey was able

465 to integrate neuronal signals from both, optogenetic and visual sources, in order to improve

466 detection performance. The response rate to catch trials, i.e. the 'false alarm rate' was low

467 (33.4\%). Interestingly, optogenetic stimulation alone was no sufficient to induce a saccadic

468 response that was significantly different from the false alarm rate (Chi-squared test; $p=0.0521$;

$469 \mathrm{n}=119$ opto only and $\mathrm{n}=467$ catch trials). Importantly, the saccade rate in the sham control

470 condition was not different from the false alarm rate (Chi-squared test; $p=0.419 ; n=116$ sham and

$471 \quad n=467$ catch trials).

472 These results show how our approach can be used to study signal integration from optogenetic

473 stimulation during perception in the visual cortex of awake behaving marmosets. 


\section{Discussion}

475 Here, we demonstrate for the first time neural recordings and optogenetic stimulation in

476 combination with behavioral manipulation in the awake behaving marmoset. Systems

477 neuroscience relies on the constant improvement of technologies for recording and manipulation

478 of neural circuits in vivo. Novel techniques, such as next-generation electrode technology, are

479 therefore being developed at a rapid pace (Steinmetz et al., 2018; Hong and Lieber, 2019).

480 Moreover, some efforts for technology development in neuroscience explicitly rely on the

481 advantages of the marmoset model (Okano et al., 2016). Motivated by these factors, we

482 implemented a novel approach that enables the use of modern neural probes in combination with

483 optogenetic stimulation and behavioral manipulation in the awake, behaving marmoset. We

484 demonstrate the functionality of our methods by obtaining multi- and single-unit recordings in two

485 visual areas simultaneously and using optogenetic stimulation to drive neural activity and 486 influence the animal's behavior in a detection task.

487 Advantages, drawbacks and further directions of the 3D printing-based

488 design

489 Our design relies heavily on the use of 3D printing technology. 3D printing allows for rapid design 490 adaptations, requires few mechanical constraints and enables the production of prototypes at low 491 cost and short turnover times (Randazzo et al., 2016; Chen et al., 2017). These factors make it 492 possible for other researchers to easily modify and improve the design presented here. There are 493 several potential adaptations that could be useful, for example: expansion of the chamber and 494 change in its position relative to the skull. Such modifications could enable recordings from more 495 lateral brain areas such as area MT or IT, which are inaccessible with the current design (Suppl. 496 Fig. 1). Moreover, the design could be adapted such that it integrates a mechanism for head 
497 fixation on the chamber (Ding et al., 2017; Johnston et al., 2018). This would make a separate

498 headpost obsolete and thereby allow better access to frontal regions. Also, the integration of a 499 head-fixation mechanism on the chamber might further enhance mechanical stability, which could 500 facilitate the use with imaging techniques.

501 One important drawback of 3D printing methods (specifically sintering methods as used here) is 502 that the untreated surface finish is rough. Therefore, additional steps are required if a highprecision fit (e.g. for headpost or screw threads) or a watertight sealing is necessary (Chen et al., 2017).

The weight of the complete implant, allowing recordings from 192 electrodes, amounted to approximately $8 \mathrm{~g}$ (Fig 2c). The titanium chamber alone weighs only $1.5 \mathrm{~g}$ and is designed to smoothly fit onto the surface of the skull with a low profile, thereby minimizing any unnecessary volume (Fig. 1a, b and 2a, b). The achieved weight minimization and the mechanical robustness of $3 \mathrm{D}$ printed titanium makes our design compatible with wireless recording technology. Dataloggers with batteries or wireless transmitters might be utilized, while remaining at an acceptable weight (Eliades and Wang, 2008; Roy and Wang, 2012; Walker et al., 2021). Importantly, the size

512 and weight of implants in head-unrestrained marmosets should remain as light as possible given that these animals can perform extremely fast head movements (Pandey et al., 2020).

\section{Semi-chronic vs. chronic and acute recordings}

515 Semi-chronic recording approaches, as presented here, do not require repeated insertions of 516 electrodes into the brain for each recording session. Thus, just like chronic recordings, they can 517 shorten the experimental preparation time and reduce the risk of infections. At the same time, 518 such an approach retains the option of moving probes deeper into the brain after signal decay or 519 in case the recording depth needs to be adjusted. The possibility to adapt recording depth is 520 especially important for target locations in deeper brain structures. Thus, semi-chronic recordings 
521 with silicon probes have been recently successfully used to record neural activity from the

522 brainstem of awake marmosets (Pomberger and Hage, 2019).

523 Yet, there are also advantages to other approaches such as chronic or acute recordings. In small

524 animals, e.g. mice, immobile, chronically implanted silicon probes can provide neural recording

525 stability over long periods of time (Okun et al., 2016; Juavinett et al., 2019; Steinmetz et al., 2021).

526 Stability is likely related to the relative absence of movement of the mouse brain inside its skull.

527 In marmosets, recent work has shown good recording stability with chronically implanted floating

528 electrode ('Utah') arrays (Walker et al., 2021). However, long term recording stability with

529 immobile silicon probes remains to be demonstrated. Furthermore, chronically implanted

530 electrode arrays, such as the 'Utah' array do not require any movable parts and can therefore be

531 completely sealed off after implantation, minimizing the risk of infections after surgery (Davis et

532 al., 2020; Walker et al., 2021). Acute recording approaches on the other hand allow for repeated

533 independent measurements and can therefore result in higher single-unit yield and make it

534 possible to quickly change recording position (Sedaghat-Nejad et al., 2019). Thus, while semi-

535 chronic recordings are advantageous in many circumstances, the individual experimental

536 requirements should be considered when evaluating different recording approaches.

537 In this work, we performed semi-chronic recordings with silicon probe technology from passive electrodes. Yet, our design is compatible with active probes such as Neuropixels (Jun et al., 2017;

Steinmetz et al., 2021) in chronic (Juavinett et al., 2019; Steinmetz et al., 2021) or semi-chronic

540 (Vöröslakos et al., 2021) configuration. Currently, electrode shanks and microdrive-mountable components of passive silicone probes as used in this work are still smaller than those of

542 Neuropixels probes (shank width: $25-50 \mu \mathrm{m}$ vs. $70 \mu \mathrm{m}$ for Neuropixels; shank thickness: $15 \mu \mathrm{m}$ vs

$54320 \mu \mathrm{m}$ for Neuropixels). However, active probes with fully integrated electronics and miniaturized

544 head stages would allow for even higher channel-count recordings and will be an important next

545 step for the advancement of neural recordings in awake marmosets. 


\section{Optogenetic manipulation of detection behavior}

547 We demonstrated the utility of our design by behavioral manipulation via optogenetic stimulation

548 of area V6 in the context of a detection task. Previous work in macaques has demonstrated that

549 optogenetic stimulation of the primary visual cortex can be readily reported via saccades (Jazayeri

550 et al., 2012; Ju et al., 2018). These findings are consistent with the view that animals perceived

551 phosphenes that were induced by optogenetic excitation of neurons in V1. In contrast, our own

552 results from area V6 indicate that optogenetic stimulation alone was not sufficient to significantly

553 modulate saccade rates. However, a clear behavioral effect was observed when laser stimulation

554 was paired with a low contrast visual stimulus. It is known from microstimulation experiments in

555 macaque $\mathrm{V} 1$ that detection sensitivity can substantially increase with behavioral training (Ni and

556 Maunsell, 2010). Furthermore, the detection of microstimulation outside of primary sensory areas

557 can require extended training (Histed et al., 2013). Similar changes in sensitivity thresholds have

558 been reported for optogenetic stimulation in the somatosensory cortex (May et al., 2014).

559 Therefore, it is plausible that further behavioral training in the marmoset would also lead to a 560 report of optogenetic stimulation alone. This aspect should be investigated in future work. 


\section{Materials and Methods}

563

564

565 each animal.

\section{Animals}

All animal experiments were approved by the responsible government office (Regierungspräsidium Darmstadt) in accordance with the German law for the protection of animals and the "European Union's Directive 2010/63/EU".

Five adult male marmosets were implanted with chamber, headpost and reference wires. Three of these animals were subsequently injected with a viral vector in area V6, and implanted with electrodes in areas V1 and V6. The decision to use male animals was due to availability and was not part of the experimental design. Table 1 lists relevant details, procedures and outcomes for

572 Table 1: List of all animals, procedures and outcomes:

\begin{tabular}{|c|c|c|c|c|c|}
\hline & Monkey A & Monkey U & Monkey D & Monkey E & Monkey P \\
\hline Sex & male & male & male & male & male \\
\hline $\begin{array}{l}\text { First surgery performed (head-post, chamber, } \\
\text { ref. wire) }\end{array}$ & yes & yes & yes & yes & yes \\
\hline Body weight at first surgery & $385 \mathrm{~g}$ & $438 \mathrm{~g}$ & $455 \mathrm{~g}$ & $530 \mathrm{~g}$ & $428 \mathrm{~g}$ \\
\hline $\begin{array}{l}\text { Second surgery performed (electrodes, viral } \\
\text { vector) }\end{array}$ & yes & yes & yes & no & no \\
\hline Body weight at second surgery & $371 \mathrm{~g}$ & $460 \mathrm{~g}$ & $445 \mathrm{~g}$ & n.a. & n.a. \\
\hline Neural recordings in $\mathrm{V} 1$ & yes & yes & yes & n.a. & n.a. \\
\hline Neural recordings in $\mathrm{V} 6$ & yes & poor & yes & n.a. & n.a. \\
\hline Optogenetic stimulation in V6 & yes & poor & yes & n.a. & n.a. \\
\hline Duration (months) after first surgery* & 40 & 26 & 26 & 26 & 26 \\
\hline Duration (months) after second surgery* & 35 & 19 & 19 & n.a. & n.a. \\
\hline Data shown in figures & $\begin{array}{l}\text { Fig.1, Fig.4, } \\
\text { Fig.5, Fig.6, } \\
\text { Fig.7, Fig.8 }\end{array}$ & - & Fig.3, Fig.7 & - & - \\
\hline
\end{tabular}

${ }^{*}$ Relative to the time this manuscript was prepared (September 2021) 


\section{Stimulus presentation}

575 Stimulus presentation was controlled by the custom-developed ARCADE toolbox

576 (https://github.com/esi-neuroscience/ARCADE), based on MATLAB (Mathworks, USA) and C++.

577 Stimuli were displayed on a TFT monitor (Samsung SyncMaster 2233RZ) at a refresh rate of

$578120 \mathrm{~Hz}$. Animals were placed at a distance of $45 \mathrm{~cm}$ to the monitor in a dimly lit recording booth.

579 A photodiode was placed in the top left corner of the monitor in order to determine exact stimulus-

580 onset times.

581 Eye tracking

582 The left eye of the animals was tracked under external infrared light illumination with a sampling 583 rate of $1 \mathrm{kHz}$ (Eyelink 1000, SR research, Canada). A $25 \mathrm{~mm} / \mathrm{F} 1.4$ lens was used at a distance 584 of $28 \mathrm{~cm}$ to the animal's eye.

\section{Implant design and 3D printing}

586 Designs were developed in Blender (www.blender.org), OnShape (https://www.onshape.com/),

587 and Solidworks (https://www.solidworks.com/). 3D renderings were generated in Blender. The

588 skull template shown in Figures 1 and 2 was segmented with 3D Slicer (https://www.slicer.org/)

589 based on high-resolution CT data from a marmoset skull archived on the MorphoSource data

590 base (https://doi.org/10.17602/M2/M5203/). Chambers and microdrive stabilizers were printed via

591 direct metal laser sintering from grade 5 (Ti6Al4V) titanium (Materialise, Belgium). Microdrives

592 were glued to the stabilizers with cyanoacrylate glue. Lids were printed via selective laser sintering

593 from PA12 nylon (Shapeways, USA). To ensure watertight sealing, a thin layer of silicone (Kwik-

594 Sil, World Precision Instruments, USA) was applied to the small ridge inside the lid that served as

595 contact area between chamber and lid. All custom implantation holders and guides were printed

596 from standard resins via stereolithography on a "Form 1" printer (Formlabs Inc., USA). Design

597 files for 3D printing can be found at https://github.com/PJendritza/Marmo/. 
598

599

600

601

602

603

604

605

606

607

608

\section{Anesthesia}

Anesthesia for all surgeries was induced with an intramuscular (i.m.) injection of a mixture of alfaxalone $(8.75 \mathrm{mg} / \mathrm{kg})$ and diazepam $(0.625 \mathrm{mg} / \mathrm{kg})$. Tramadol $(1.5 \mathrm{mg} / \mathrm{kg})$ and metamizol (80 $\mathrm{mg} / \mathrm{kg}$ ) were injected i.m. for initial analgesic coverage. Subsequently, a continuous intravenous (i.v.) infusion was provided through the lateral tail vein. The i.v. mixture contained glucose, amino acids (Aminomix 1 Novum, Fresenius Kabi, Germany), dexamethasone $(0.2-0.4 \mathrm{mg} \cdot \mathrm{kg}-1 \cdot \mathrm{h}-1)$, tramadol $(0.5-1.0 \mathrm{mg} \cdot \mathrm{kg}-1 \cdot \mathrm{h}-1)$ and metamizol $(20-40 \mathrm{mg} \cdot \mathrm{kg}-1 \cdot \mathrm{h}-1)$. The maximal infusion rate was $5 \mathrm{ml} \cdot \mathrm{kg}-1 \cdot \mathrm{h}-1$. Animals were breathing spontaneously throughout the surgery via a custom 3D printed face mask that applied isoflurane (0.5-2\% in $100 \%$ oxygen). Heart rate, respiration rate and body temperature were constantly monitored (Model 1030 Monitoring Gating System, SAll, USA).

\section{Implantation of chamber and headpost}

After placing the animal in a stereotaxic apparatus for the first surgery, an incision was made on the dorsal part of the skull. The temporal muscle was slightly retracted $(<5 \mathrm{~mm}$ from the superior temporal lines) and all soft tissue was completely removed from the bone surface. The bone was first cleaned by mechanical abrasion, then scrubbed with $5 \% \mathrm{H}_{2} \mathrm{O}_{2}$ and rinsed with saline. For an optimal bonding between cement and bone, the skull surface was roughened with a metal brush, and any remaining dust was removed. After the bone was completely clean and dry, we applied a thin layer of light-curable dental adhesive (All-Bond Universal, BISCO). After drying and curing with blue light, we applied a thin layer $(<1 \mathrm{~mm})$ of dental cement on top of the adhesive. Once the cement was cured, a small bur hole was drilled just anterior of the chamber. Two platinum wires (PT-5T, Science Products) were implanted epidurally at this location and served as backup reference wires for the recordings (the actual reference wires were later implanted subdurally in the second surgery). 


\section{Injection of the viral vector}

623 Viral vectors (AAV1.CamKIla.Chronos-eYFP-WPRE) were injected with a microinjector pump 624 (UMP3-1, World Precision Instruments), holding a 10uL microsyringe (NanoFil syringe, World 625 Precision Instruments) to which a 35G injection needle was attached. A durotomy of approx. $6261.5 \mathrm{~mm}$ was performed with a bent $25 \mathrm{G}$ cannula, and the vector was injected at two depths $627(-1.4 \mathrm{~mm}$ and $-0.5 \mathrm{~mm}$ from the surface). A volume of $2.5 \mu \mathrm{l}$ at each depth was injected at a speed of $200 \mathrm{~nL} / \mathrm{min}$ (total injected volume $=5.0 \mu \mathrm{l}$ ). To ensure sufficient diffusion of the viral vector, we waited 10 min after the each injection before moving or retracting the needle.

\section{Silicon probes}

631 Silicon probes were semi-chronically implanted in areas V1 and V6, mounted on one microdrive 632 per area (Nano-Drive CN-01 V1, Cambridge NeuroTech, UK). Two 32-channel shanks with $633250 \mu \mathrm{m}$ spacing were implanted in V1, and four 32-channel shanks in V6 (H2 probe, Cambridge 634 NeuroTech, UK). Electrode implantation was performed directly following the injection of the viral 635 vector. Electrode tips were disinfected shortly before the implantation by dipping them twice in $63670 \%$ ethanol for $45 \mathrm{~s}$. After the electrodes were in place and the cement was hardened, craniotomies were sealed by applying several drops of soft silicone gel (DOWSIL 3-4680, Dow Corning).

\section{Acquisition and processing of neural data}

640 Neural signals were recorded through active, unity gain head stages (ZC32, Tucker Davis 641 Technologies, USA), digitized at 24,414.0625 Hz (PZ2 preamplifier, Tucker Davis Technologies, 642 USA) and re-sampled offline to $25 \mathrm{kHz}$. Sample-by-sample re-referencing was applied by 643 calculating the median across all channels for each shank and subtracting this signal from each 644 channel of the corresponding shank (Jun et al., 2017). Data was band-pass filtered for spiking 645 activity either with a 4th-order Butterworth filter $(0.3-6 \mathrm{kHz})$ or, in case optogenetic stimulation 
646

647

648

649

650

\section{Optogenetic stimulation}

652 Optogenetic stimulation was performed with a laser beam combiner (LightHUB, Omicron laserage), housing a $100 \mathrm{~mW}$ diode laser with a wavelength of $505 \mathrm{~nm}$ (LuxXplus 505-100) with direct modulation and a $100 \mathrm{~mW}$ DPSS laser with a wavelength of $594 \mathrm{~nm}$ (OBIS 594-100) with direct modulation. The combined lasers were coupled to a $50 \mu \mathrm{m} / 0.22 \mathrm{NA}$ optic fiber which was connected to a fiber optic cannula $(200 \mu \mathrm{m}$ core diameter, $0.39 \mathrm{NA}$, Doric Lenses Inc.). The cannula was held by a micromanipulator (SM-25C, Narishige) and was positioned approx. $4 \mathrm{~mm}$ above the craniotomy during recording/stimulation sessions. Laser power was calibrated prior to

659 the experiments with a photodiode-based optical power meter (PM130D, Thorlabs). Output power 660 was measured at the tip of the fiber optic cannula. Laser waveforms were generated by a real661 time signal processor (RZ2 bioamp processor, Tucker Davis Technologies, USA). To avoid 662 artifacts arising from sharp transients in laser intensity (Cardin et al., 2010), we only used smooth on and offsets (Wu et al., 2015). This was done by using one half of a sine wave as a taper at the 664 beginning and end of any sharp signal (5 ms trough-to-peak time, with the trough having an 665 intensity of $0 \mathrm{~mW}$ ).

\section{CT scans and segmentation}

667 CT scans were performed under brief anesthesia induced with an intramuscular (i.m.) injection of 668 a mixture of alfaxalone $(8.75 \mathrm{mg} / \mathrm{kg})$ and diazepam $(0.625 \mathrm{mg} / \mathrm{kg})$. The head of the animal was 669 stabilized via the headpost for the duration of the scan. CTs were performed with a Planmeca Jendritza et al. 
670 ProMax 3D Mid scanner (Planmeca Oy, Finland) at $90 \mathrm{kV}$ and $10 \mathrm{~mA}$ with a voxel size of $150 \mu \mathrm{m}$

671 (isotropic). Segmentation of CT data was performed with 3D Slicer. Models were exported as STL

672 files and imported into Blender for alignment.

673 Spike sorting and single unit analysis

674 Spike sorting was performed offline with Kilosort (Pachitariu et al., 2016). Average spike 675 waveforms were calculated from the trimmed mean (5\% outlier exclusion). Autocorrelation 676 functions were generated at a resolution of $0.33 \mathrm{~ms}$ and normalized by dividing by the maximum 677 value after removal of the central peak.

678 Receptive field mapping

679 All details about the RF mapping procedure have been described previously in Jendritza et al., 680 2021. RF mapping was performed with stimuli consisting of black wedges and annuli of various 681 orientations and sizes, presented on a gray background for a duration of eight frames $(120 \mathrm{~Hz}$ 682 monitor refresh rate). For RF calculation, MUA data was cut into epochs of $280 \mathrm{~ms}$ (from $100 \mathrm{~ms}$ 683 before to $180 \mathrm{~ms}$ after stimulus onset). Epochs were included in the analysis if the eye position 684 remained inside the fixation window throughout the epoch. For noise-rejection purposes, we 685 excluded epochs in which the standard deviation of MUA across time was more than 10-times 686 larger than the median standard deviation across all epochs. Sites were considered to be modulated if the mean MUA from at least three different wedge stimuli and at least three different annulus stimuli evoked a response that was significantly larger (paired t-test, alpha $=0.01$ ) than the MUA during baseline (100 ms to $0 \mathrm{~ms}$ prior to stimulus onset). For plotting, MUA was normalized per site to have a value between zero and one. RF plots and outlines were generated by truncating the normalized MUA at a value of 0.2 . 


\section{Passive fixation task}

693 A passive fixation task was used to measure neural responses following visual stimulation with 694 gratings. At the beginning of each trial, the animal was required to maintain its gaze at a central 695 fixation point within a window of $1.4^{\circ}$ radius for $100-120 \mathrm{~ms}$. After this period, a static square696 wave grating was presented for $650 \mathrm{~ms}$ at a Michelson contrast of $80 \%$. The size and orientation of the grating was selected at random for each trial. Possible values for the grating radius (in degrees of visual angle) were: $5^{\circ}, 7.25^{\circ}, 9.5^{\circ}, 11.75^{\circ}$ and $14^{\circ}$. Possible values for the grating orientation were: $22.5^{\circ}, 45^{\circ}, 67.5^{\circ}, 90^{\circ}, 112.5^{\circ}, 135^{\circ}, 157.5^{\circ}$ and $180^{\circ}$. After stimulus offset, the animal was required to maintain it gaze in the fixation widow for another $100 \mathrm{~ms}$. After a correct trial, a picture of a marmoset face was displayed in the center of the monitor, and the animal was rewarded. The amount of reward was $0.07 \mathrm{ml}$ per trial at the start of the session and increased by $0.02 \mathrm{ml}$ for every $10 \mathrm{ml}$ consumed (capped at $0.1 \mathrm{ml}$ per trial). Reward was provided via a lick spout and consisted of diluted gum arabic.

\section{Visual and optogenetic detection task}

706 At the beginning of each trial of the detection task, the animal was required to position its gaze at 707 a central fixation point within a window of $1.5^{\circ}$ radius for $100-150 \mathrm{~ms}$. After this period, a 708 background stimulus was presented, while the monkey maintained fixation. The background stimulus was a full-screen circular grating, concentric to the fixation point and either contracting towards or expanding from the fixation point, each in a random half of the trials (contrast $=40 \%$, spatial freq. $=2 \mathrm{cycles}^{\circ}$, temporal freq. $=1$ cycle/s). At $150-320 \mathrm{~ms}$ after the onset of the background stimulus, a black, moving circular patch $\left(1.8^{\circ}\right.$ diameter, moving at $5.74 \%$, linear motion, random direction) with either high (50\%) contrast or low contrast $(7.8 \%)$ was presented

714 for $250 \mathrm{~ms}$. The center of the movement path of the circular patch was fixed in the lower right 715 quadrant, where the receptive fields of the optogenetically responsive V6 cells were located. 
717

718

719

720

721

722

723

724

725

726

727

728

729

730

731

732

733

734

735

736

737

738

739

740

741 absence of a visual target. Furthermore, a control "sham" stimulation condition was included, with sham trials being identical to real optogenetic stimulation trials (without visual target), but with the laser output switched to a second optic fiber that was placed $2 \mathrm{~mm}$ outside the craniotomy. All of these "go" trials (60\% of all trials) were categorized as hits if the animal made a saccade away from the fixation point within $500 \mathrm{~ms}$ after the onset of the moving circular patch or the laser. Responses that were faster than $50 \mathrm{~ms}$ were categorized as early responses and were not rewarded. $50 \%$ of trials with a visible target were coupled with optogenetic stimulation that consisted of a $250 \mathrm{~ms}$ square pulse with an amplitude of $25 \mathrm{~mW}$. The onset timing for visual and optogenetic stimulation was determined by the computer controlling the visual stimulation. We did not compensate for any delay between trigger onset and actual onset of the visual stimulus on the monitor. In the remaining "catch" trials ( $40 \%$ of all trials), no visual or optogenetic target was presented, and the monkey was rewarded for maintaining its gaze at the fixation point for 800 ms. After a correct saccade, or a correct rejection (maintained fixation), a picture of a marmoset face was displayed in the center of the monitor, and the animal was rewarded. The amount of reward was $0.0625 \mathrm{ml}$ per trial at the start of the session and increased by $0.02 \mathrm{ml}$ for every $10 \mathrm{ml}$ consumed (capped at $0.1 \mathrm{ml}$ per trial).

In the detection task described above, catch trials were longer than the average go trial. Thus, simply calculating saccade rates from catch trials would lead to an overestimation of the true false alarm rate, because the monkey had more time to perform a saccade in a catch trial than in a go trial. False-alarm rate calculation was therefore performed in the following way: One randomly selected catch trial with false alarm was compared with the timing of a randomly selected go-trial. If the time of the false alarm from the selected catch trial fell within the time window in which the monkey would have performed a hit, the trial was categorized as a false alarm. If the false alarm timing was such that the monkey would have missed the target, the trial was categorized as correct rejection. This random pairing was performed for $n=467$ random pairs of trials, as this was

Jendritza et al. 
742 the expected number of catch trials ( $40 \%$ of all trials), given the total number of hits and misses

743 performed by the animal $(n=700)$. The proportion of false alarms and the respective binomial

744 confidence intervals were then calculated for this random sample. This procedure was repeated

7451000 times, and the false-alarm rates and confidence intervals from all shuffling iterations were

746 averaged.

\section{Acknowledgements}

748 We thank Marianne Hartmann for her constant support in training the animals, Martin Vinck for 749 providing access to his GPU systems and Gustavo Rohenkohl for his feedback on the manuscript.

750 This work was supported by DFG (SPP 1665 FR2557/1-1, FOR 1847 FR2557/2-1, FR2557/5-1-

751 CORNET, FR2557/6-1-NeuroTMR, FR2557/7-1-DualStreams to P.F.), EU (HEALTH-F2-2008-

752 200728-BrainSynch, FP7-604102-HBP, FP7-600730-Magnetrodes to P.F.), a European Young

753 Investigator Award to P.F., National Institutes of Health (1U54MH091657-WU-Minn-Consortium-

754 HCP to P.F.), the LOEWE program (NeFF to P.F.).

\section{Declaration of interests}

756 P.F. has a patent on thin-film electrodes and is beneficiary of a respective license contract with

757 Blackrock Microsystems LLC (Salt Lake City, UT, USA). P.F. is a member of the Scientific

758 Technical Advisory Board of CorTec GmbH (Freiburg, Germany) and is managing director of 759 Brain Science GmbH (Frankfurt am Main, Germany). 


\section{References}

Cardin JA, Carlén M, Meletis K, Knoblich U, Zhang F, Deisseroth K, Tsai LH, Moore CI (2010) Targeted optogenetic stimulation and recording of neurons in vivo using cell-type-specific expression of Channelrhodopsin-2. Nat Protoc.

Chen R, Gore F, Nguyen QA, Ramakrishnan C, Patel S, Kim SH, Raffiee M, Kim YS, Hsueh B, Krook-Magnusson E, Soltesz I, Deisseroth K (2021) Deep brain optogenetics without intracranial surgery. Nat Biotechnol.

Chen X, Possel JK, Wacongne C, van Ham AF, Klink PC, Roelfsema PR (2017) 3D printing and modelling of customized implants and surgical guides for non-human primates. J Neurosci Methods.

Courellis HS, Nummela SU, Metke M, Diehl GW, Bussell R, Cauwenberghs G, Miller CT (2019) Spatial encoding in primate hippocampus during free navigation. PLoS Biol.

Davis ZW, Muller L, Martinez-Trujillo J, Sejnowski T, Reynolds JH (2020) Spontaneous travelling cortical waves gate perception in behaving primates. Nature.

Deisseroth K (2015) Optogenetics: 10 years of microbial opsins in neuroscience. Nat Neurosci.

Ding R, Liao X, Li J, Zhang J, Wang M, Guang Y, Qin H, Li X, Zhang K, Liang S, Guan J, Lou J, Jia $\mathrm{H}$, Chen B, Shen H, Chen X (2017) Targeted patching and dendritic Ca2+ imaging in nonhuman primate brain in vivo. Sci Rep.

Ebina T, Obara K, Watakabe A, Masamizu Y, Terada SI, Matoba R, Takaji M, Hatanaka N, Nambu A, Mizukami H, Yamamori T, Matsuzaki M (2019) Arm movements induced by noninvasive optogenetic stimulation of the motor cortex in the common marmoset. Proc Natl Acad Sci U S A.

Eliades SJ, Wang X (2008) Chronic multi-electrode neural recording in free-roaming monkeys. J Neurosci Methods.

Fenno L, Yizhar O, Deisseroth K (2011) The development and application of optogenetics. Annu Rev Neurosci.

Histed MH, Ni AM, Maunsell JHR (2013) Insights into cortical mechanisms of behavior from microstimulation experiments. Prog Neurobiol 103:115-130.

Hong G, Lieber CM (2019) Novel electrode technologies for neural recordings. Nat Rev Neurosci.

Jackson N, Muthuswamy J (2008) Artificial dural sealant that allows multiple penetrations of implantable brain probes. J Neurosci Methods.

Jazayeri M, Lindbloom-Brown Z, Horwitz GD (2012) Saccadic eye movements evoked by optogenetic activation of primate V1. Nat Neurosci.

Jendritza P, Klein FJ, Rohenkohl G, Fries P (2021) Visual neuroscience methods for marmosets: Efficient receptive field mapping and head-free eye tracking. eNeuro.

Johnston K, Ma L, Schaeffer L, Everling S (2019) Alpha oscillations modulate preparatory activity in marmoset area $8 \mathrm{Ad}$. J Neurosci. 
Johnston KD, Barker K, Schaeffer L, Schaeffer D, Everling S (2018) Methods for chair restraint and training of the common marmoset on oculomotor tasks. J Neurophysiol.

Ju N, Jiang R, Macknik SL, Martinez-Conde S, Tang S (2018) Long-term all-optical interrogation of cortical neurons in awake-behaving nonhuman primates. PLoS Biol.

Juavinett AL, Bekheet G, Churchland AK (2019) Chronically implanted neuropixels probes enable high-yield recordings in freely moving mice. Elife.

Jun JJ et al. (2017) Fully integrated silicon probes for high-density recording of neural activity. Nature.

Klapoetke NC et al. (2014) Independent optical excitation of distinct neural populations. Nat Methods 11.

Komatsu M, Sugano E, Tomita H, Fujii N (2017) A chronically implantable bidirectional neural interface for non-human primates. Front Neurosci.

Kondo T, Saito R, Otaka M, Yoshino-Saito K, Yamanaka A, Yamamori T, Watakabe A, Mizukami H, Schnitzer MJ, Tanaka KF, Ushiba J, Okano H (2018) Calcium Transient Dynamics of Neural Ensembles in the Primary Motor Cortex of Naturally Behaving Monkeys. Cell Rep.

Liu C, Ye FQ, Yen CCC, Newman JD, Glen D, Leopold DA, Silva AC (2018) A digital 3D atlas of the marmoset brain based on multi-modal MRI. Neuroimage.

Lui LL, Bourne JA, Rosa MGP (2006) Functional response properties of neurons in the dorsomedial visual area of New World monkeys (Callithrix jacchus). Cereb Cortex 16:162177.

Macdougall M, Nummela SU, Coop S, Disney A, Mitchell JF, Miller CT (2016) Optogenetic manipulation of neural circuits in awake marmosets. J Neurophysiol.

May T, Ozden I, Brush B, Borton D, Wagner F, Agha N, Sheinberg DL, Nurmikko A V. (2014) Detection of optogenetic stimulation in somatosensory cortex by non-human primates Towards artificial tactile sensation. PLoS One.

Mehta P, Kreeger L, Wylie DC, Pattadkal JJ, Lusignan T, Davis MJ, Turi GF, Li WK, Whitmire MP, Chen Y, Kajs BL, Seidemann E, Priebe NJ, Losonczy A, Zemelman B V. (2019) Functional Access to Neuron Subclasses in Rodent and Primate Forebrain. Cell Rep.

Miller CT (2017) Why marmosets? Dev Neurobiol.

Miller CT, Freiwald WA, Leopold DA, Mitchell JF, Silva AC, Wang X (2016) Marmosets: A Neuroscientific Model of Human Social Behavior. Neuron 90:219-233.

Mitchell JF, Leopold DA (2015) The marmoset monkey as a model for visual neuroscience. Neurosci Res.

Ni AM, Maunsell JHR (2010) Microstimulation Reveals Limits in Detecting Different Signals from a Local Cortical Region. Curr Biol.

O'Connor HJ, Dickson AN, Dowling DP (2018) Evaluation of the mechanical performance of polymer parts fabricated using a production scale multi jet fusion printing process. Addit Manuf.

Okano H (2021) Current Status of and Perspectives on the Application of Marmosets in 
Neurobiology. Annu Rev Neurosci.

Okano H, Sasaki E, Yamamori T, Iriki A, Shimogori T, Yamaguchi Y, Kasai K, Miyawaki A (2016) Brain/MINDS: A Japanese National Brain Project for Marmoset Neuroscience. Neuron.

Okun M, Lak A, Carandini M, Harris KD (2016) Long term recordings with immobile silicon probes in the mouse cortex. PLoS One.

Pachitariu M, Steinmetz N, Kadir S, Carandini M, Kenneth D. H (2016) Kilosort: realtime spikesorting for extracellular electrophysiology with hundreds of channels. bioRxiv.

Pandey S, Simhadri S, Zhou Y (2020) Rapid Head Movements in Common Marmoset Monkeys. iScience 23:100837.

Panzeri S, Macke JH, Gross J, Kayser C (2015) Neural population coding: Combining insights from microscopic and mass signals. Trends Cogn Sci.

Paxinos G, Watson C, Petrides M, Rosa M, Tokuno H (2012) The marmoset brain in stereotaxic coordinates. Elsevier Academic Press.

Poggio T (2011) The computational magic of the ventral stream. Nat Preced.

Pomberger T, Hage SR (2019) Semi-chronic laminar recordings in the brainstem of behaving marmoset monkeys. J Neurosci Methods.

Porada I, Bondar I, Spatz WB, Krüger J (2000) Rabbit and monkey visual cortex: More than a year of recording with up to 64 microelectrodes. J Neurosci Methods.

Randazzo M, Pisapia J, Singh N, Thawani J (2016) 3D printing in neurosurgery: A systematic review. Surg Neurol Int.

Remington ED, Osmanski MS, Wang X (2012) An Operant Conditioning Method for Studying Auditory Behaviors in Marmoset Monkeys. PLoS One.

Roy S, Wang X (2012) Wireless multi-channel single unit recording in freely moving and vocalizing primates. J Neurosci Methods.

Sasaki E et al. (2009) Generation of transgenic non-human primates with germline transmission. Nature.

Sato K, Sasaguri H, Kumita W, Inoue T, Kurotaki Y, Nagata K, Mihira N, Sato K, Sakuma T, Yamamoto T, Tagami M, Manabe R, Ozaki K, Okazaki Y, Saido TC, Sasaki E (2020) A non-human primate model of familial Alzheimer's disease. bioRxiv.

Saxena S, Cunningham JP (2019) Towards the neural population doctrine. Curr Opin Neurobiol.

Sedaghat-Nejad E, Herzfeld DJ, Hage P, Karbasi K, Palin T, Wang X, Shadmehr R (2019) Behavioral training of marmosets and electrophysiological recording from the cerebellum. $J$ Neurophysiol.

Servick K (2018) Why are U.S. neuroscientists clamoring for marmosets? Science (80- ):383384.

Shobe JL, Claar LD, Parhami S, Bakhurin KI, Masmanidis SC (2015) Brain activity mapping at multiple scales with silicon microprobes containing 1,024 electrodes. J Neurophysiol. 
Solomon SG, Rosa MGP (2014) A simpler primate brain: The visual system of the marmoset monkey. Front Neural Circuits.

Steinmetz NA et al. (2021) Neuropixels 2.0: A miniaturized high-density probe for stable, longterm brain recordings. Science (80-).

Steinmetz NA, Koch C, Harris KD, Carandini M (2018) Challenges and opportunities for largescale electrophysiology with Neuropixels probes. Curr Opin Neurobiol.

Stevenson MF, Poole TB (1976) An ethogram of the common marmoset (Calithrix jacchus jacchus): General behavioural repertoire. Anim Behav 24:428-451.

Tomioka I, Nogami N, Nakatani T, Owari K, Fujita N, Motohashi H, Takayama O, Takae K, Nagai Y, Seki K (2017) Generation of transgenic marmosets using a tetracyclin-inducible transgene expression system as a neurodegenerative disease model. Biol Reprod.

Vöröslakos M, Petersen PC, Vöröslakos B, Buzsáki G (2021) Metal microdrive and head cap system for silicon probe recovery in freely moving rodent. Elife.

Walker JD, Pirschel F, Sundiang M, Niekrasz M, MacLean JN, Hatsopoulos NG (2021) Chronic wireless neural population recordings with common marmosets. Cell Rep.

Wolff SB, Ölveczky BP (2018) The promise and perils of causal circuit manipulations. Curr Opin Neurobiol.

Wu F, Stark E, Ku PC, Wise KD, Buzsáki G, Yoon E (2015) Monolithically Integrated $\mu$ LDs on Silicon Neural Probes for High-Resolution Optogenetic Studies in Behaving Animals. Neuron.

Yamada Y, Matsumoto Y, Okahara N, Mikoshiba K (2016) Chronic multiscale imaging of neuronal activity in the awake common marmoset. Sci Rep.

Yu HH, Rosa MGP (2014) Uniformity and diversity of response properties of neurons in the primary visual cortex: Selectivity for orientation, direction of motion, and stimulus size from center to far periphery. Vis Neurosci. 


\section{Supplementary Figures}

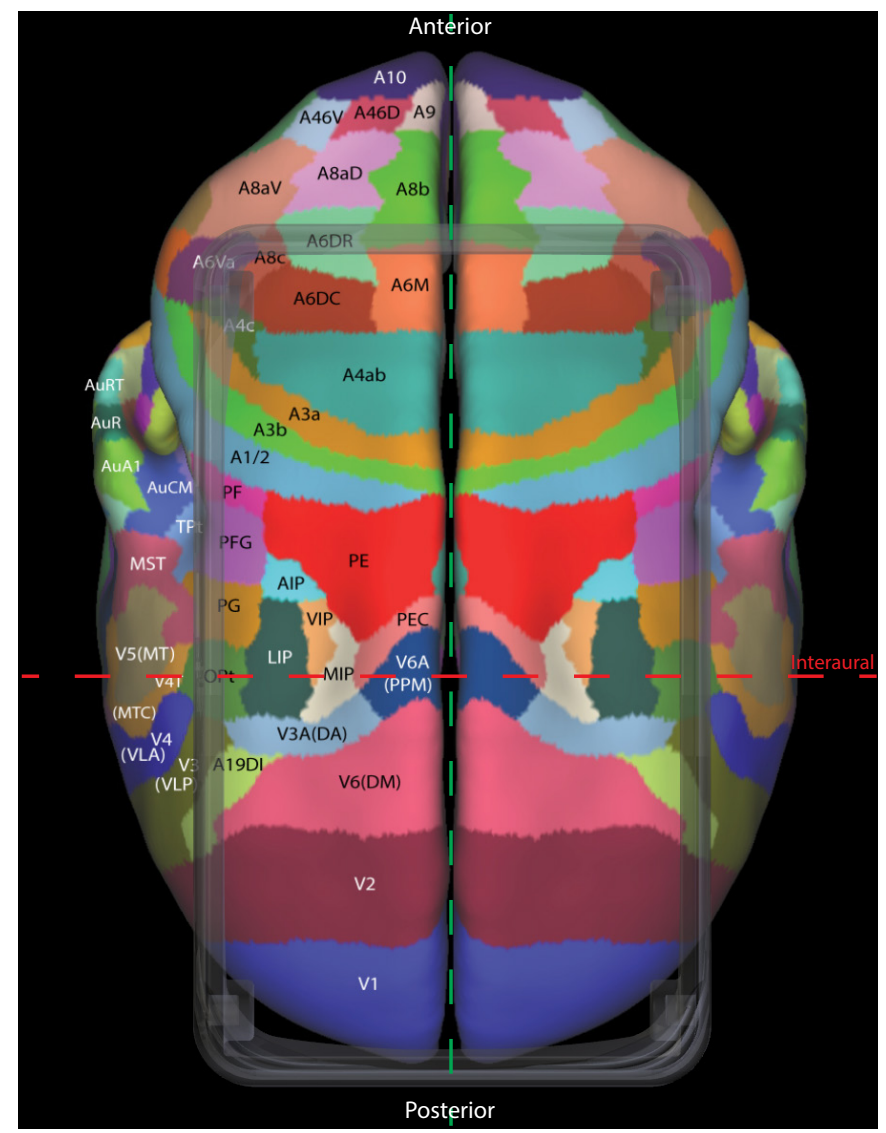

904 Supplementary Figure 1| Accessible cortical areas. Top view of the chamber and cortical brain areas directly

905 underneath. Red dashed line indicates interaural axis. Green dashed line indicates anterior-posterior axis. Area

906 segmentation and labels from Paxinos et al. (2012). 
bioRxiv preprint doi: https://doi.org/10.1101/2021.10.30.466578; this version posted November 2, 2021. The copyright holder for this preprint (which was not certified by peer review) is the author/funder, who has granted bioRxiv a license to display the preprint in perpetuity. It is made available under aCC-BY 4.0 International license.

a)

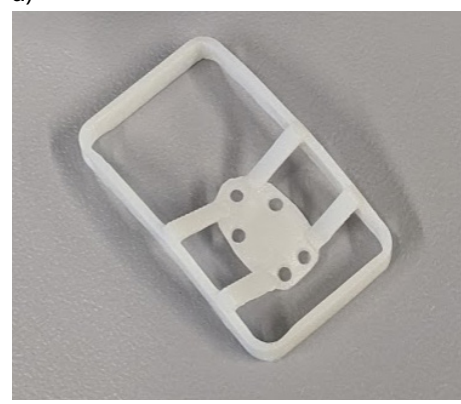

b)

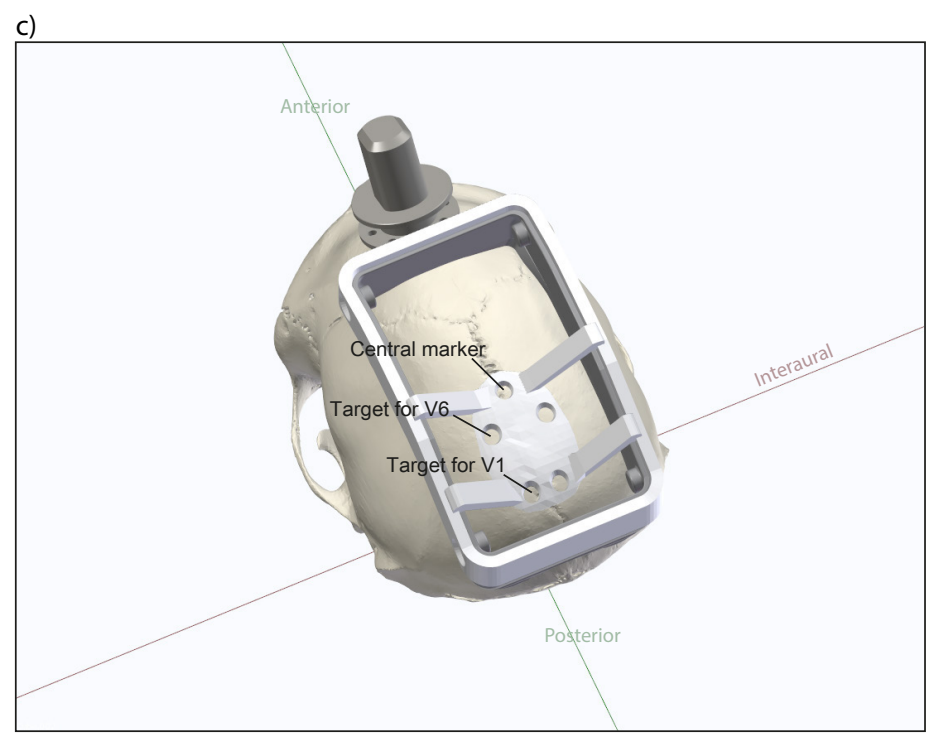

908 Supplementary Figure 2 | 3D printed implantation target guide. Photograph of the implantation guide (a) before

909 and (b) after placement on the chamber. c) 3D rendering of the implantation guide placed on the chamber. The guide

910 hole for the central marker indicates the anterior-posterior and medio-lateral center of the stereotaxic coordinate

911 system. Guide holes for areas V1 and V6 are indicated for the left hemisphere. 